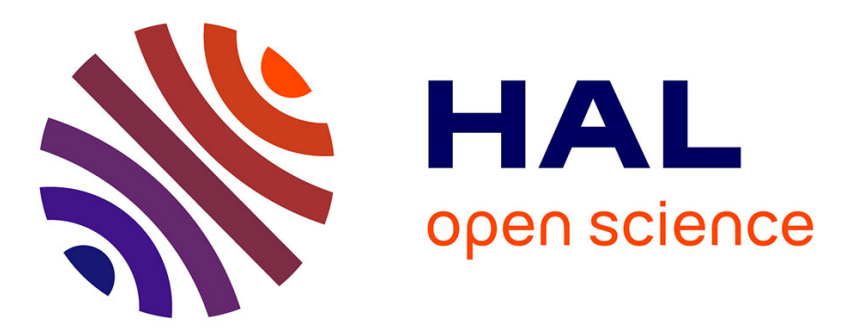

\title{
Nonlinear axisymmetric deformations of an elastic tube under external pressure
}

Yunfei Zhu, Xiaoyu Luo, Raymond Ogden

\section{To cite this version:}

Yunfei Zhu, Xiaoyu Luo, Raymond Ogden. Nonlinear axisymmetric deformations of an elastic tube under external pressure. European Journal of Mechanics - A/Solids, 2010, 29 (2), pp.216-229. 10.1016/j.euromechsol.2009.10.004 . hal-01303324

\section{HAL Id: hal-01303324 \\ https://hal.science/hal-01303324}

Submitted on 18 Apr 2016

HAL is a multi-disciplinary open access archive for the deposit and dissemination of scientific research documents, whether they are published or not. The documents may come from teaching and research institutions in France or abroad, or from public or private research centers.
L'archive ouverte pluridisciplinaire HAL, est destinée au dépôt et à la diffusion de documents scientifiques de niveau recherche, publiés ou non, émanant des établissements d'enseignement et de recherche français ou étrangers, des laboratoires publics ou privés. 


\title{
Nonlinear axisymmetric deformations of an elastic tube under external pressure
}

\author{
Y. Zhu, X.Y. Luo, R.W. Ogden \\ Department of Mathematics, University of Glasgow, Glasgow G12 8QW, UK
}

\begin{abstract}
The problem of the finite axisymmetric deformation of a thick-walled circular cylindrical elastic tube subject to pressure on its external lateral boundaries and zero displacement on its ends is formulated for an incompressible isotropic neo-Hookean material. The formulation is fully nonlinear and can accom-modate large strains and large displacements. The governing system of nonlinear partial differential equations is derived and then solved numerically using the $\mathrm{C}++$ based object-oriented finite element library Libmesh. The weighted residual-Galerkin method and the Newton-Krylov nonlinear solver are adopted for solving the governing equations. Since the nonlinear problem is highly sensitive to small changes in the numerical scheme, convergence was obtained only when the analytical Jacobian matrix was used. A Lagrangian mesh is used to discretize the governing partial differential equations. Results are presented for different parameters, such as wall thickness and aspect ratio, and comparison is made with the corresponding linear elasticity formulation of the problem, the results of which agree with those of the nonlinear formulation only for small external pressure. Not surprisingly, the nonlinear results depart significantly from the linear ones for larger values of the pressure and when the strains in the tube wall become large. Typical nonlinear characteristics exhibited are the "corner bulging" of short tubes, and multiple modes of deformation for longer tubes.
\end{abstract}

Nonlinear elasticity, Finite deformation, Large strain, Elastic tubes, Axisymmetric deformations

\section{Introduction}

In many engineering and biomechanical applications cylindrical tubes are subject to external pressures and as a result undergo large (nonlinear) deformations. In early engineering approaches to the analysis of this problem it was typically assumed that the material response is linearly elastic, but this led to predictions which were inaccurate except for very small deformations. It is well known that for biological materials deformations of the order $50-100 \%$ can occur, and in this case a fully nonlinear problem formulation is essential. However, fully nonlinear material and geometrical analysis is challenging due to the difficulty of solving such problems. To facilitate solutions simplifications are often made, such as the adoption of thin shell theories, which have been successful for describing thin-walled structures (Yamaki, 1969, 1984; Libai and Simmonds, 1998). Some researchers have focused on geometrically nonlinear problems, with small strains but large displacements, and this approach has often proved to be adequate. Erbay and Demiray (1995) considered the finite axisymmetric deformation of a circular cylindrical tube of neo-Hookean material by using an asymptotic expansion method. Their perturbation solution is based on the smallness of the ratio of thickness to inner radius of the tube. Normal and tangential tractions were applied on the inner surface of the tube but no boundary conditions were considered at the ends of the tube. Heil and Pedley (1995) and Marzo et al. (2005) performed a numerical simulation of the post-buckling behaviour of tubes under external pressure.

Propagation of finite amplitude waves in fluid-filled elastic or viscoelastic thin-walled tubes has been investigated by Anlike et al (1968); Rudinger (1970); Moodie and Swaters (1989) and Erbay and Demiray (1995). However, for thick-walled tubes there are few results available in the literature due to the difficulties arising from the variation of field quantities with the radial coordinate. Demiray studied weakly nonlinear waves in a fluid filled thick-walled elastic tube, first using an artificial estimated pressure dependence (Demiray, 1996) on the axial coordinate, which was later improved upon (Demiray, 1998).

The ability to predict the bifurcation character of the solutions is also an important practical problem. Negrón-Marrero (1999) studied the bifurcation of axisymmetric hyperelastic cylinders subject to nonlinear mixed boundary conditions and found that the eigenfunctions can be classified into those that are symmetric about the mid-plane, representing either necked or barrelled configurations of the cylinder, and those that break this symmetry. Finite axisymmetric deformations of thick-walled carbon-black filled rubber tubes were also studied experimentally by Beatty and 
Dadras (1976). They found that for aspect ratios less than 5 tubes exhibit radially or axially symmetric bulging modes of deformation, distinct from the familiar Euler buckling that occurs for longer tubes. Significantly, they found that the experimentally observed critical compression load is considerably lower than that predicted on the basis of the linear theory.

For problems involving finite elastic deformations, a rigorous bifurcation theory has been established based on the analysis of infinitesimal deformations superimposed on a known large deformation (Green et al., 1952). Using this theory, Nowinski and Shahinpoor (1969) examined the stability of an infinitely long circular cylinder of neo-Hookean material under external pressure assuming a plane strain deformation, and Wang and Ertepinar (1972) investigated the stability of infinitely long cylindrical shells and spherical shells subjected to both internal and external pressure. On the same basis, but for different (incompressible, isotropic) material models, Haughton and Ogden (1979) examined in some detail the bifurcation behaviour of circular cylindrical tubes of finite length under internal pressure and axial loading.

Bifurcation from a circular cylindrical configuration of a thickwalled tube subject to combined axial loading and external pressure was investigated on the basis of the nonlinear theory of elasticity by Zhu et al. (2008). Their work showed that the wall thickness and aspect ratio play important roles in the occurrence of the most unstable bifurcation mode. Different from the results based on thin shell theories, which show that higher modes should occur for shorter tubes, Zhu et al. (2008) showed that mode- 2 becomes more persistent for shorter tubes if a suitable nonlinear model is used. This observation was in agreement with experimental findings on thick-walled tubes subject to external pressure, in particular those of Bertram $(1982,1987)$ and Bertram et al. (1990). However, a limitation of this work is that the bifurcation analysis was initiated from a deformed circular cylindrical configuration of an elastic tube with rather special incremental boundary conditions imposed on the ends of the tube. Thus, the results only apply for the initial bifurcation behaviour, and might preclude realistic post-buckling behaviour involving large displacements near the ends of the tube.

In the present paper, we formulate the fully nonlinear problem of the large axisymmetric deformations of thick-walled cylindrical tubes of finite length made of incompressible hyperelastic material subject to zero displacements on the ends of the tube and hydrostatic pressure on the exterior of the lateral surface. The general governing differential equations that describe the deformation of the tube are derived, with both geometrical and material nonlinearity included. The corresponding radially-symmetric and linear problems are also examined for the purpose of comparison. The sets of equations are solved numerically using the object-oriented $\mathrm{C}++$ finite element package Libmesh. Results for tubes with different aspect ratios are presented to show how the wall thickness and tube length affect the nonlinear behaviour. The major findings are that for a short tube with smaller aspect ratio, the nonlinear deformation is characterized by a corner bulging, which changes all the stress distributions, especially for the shear stress. For longer tubes, the nonlinear model exhibits higher modes of deformation while for the corresponding linear model only mode-2 is present. The agreement between the linear and nonlinear models is only good for small values of the pressure, corresponding to maximum strains of about $5 \%$.

\section{Basic equations}

We consider an initially stress-free thick-walled circular cylindrical tube. In this reference configuration the geometry of the tube is described in terms of cylindrical polar coordinates $R, \Theta, Z$ by
$A \leq R \leq B, \quad 0 \leq \Theta \leq 2 \pi, \quad 0 \leq Z \leq L$

where $A$ and $B$, respectively, are the inner and outer radii and $L$ is the length of the tube. Let $\mathbf{E}_{R}, \mathbf{E}_{\Theta}, \mathbf{E}_{Z}$ denote the associated unit basis vectors. The deformed geometry is described in terms of cylindrical polar coordinates $r, \theta, z$ with corresponding unit basis vectors $\mathbf{e}_{r}, \mathbf{e}_{\theta}$, $\mathbf{e}_{z}$. In what follows we shall consider axisymmetric deformations of the tube.

\subsection{Deformation}

Let $\mathbf{X}, \mathbf{x}$ denote the position vectors of an arbitrary material point in the reference and deformed configurations, respectively. We write

$\mathbf{x}=\mathbf{X}+\mathbf{u}$

where $\mathbf{u}$ is the displacement vector, which, for axisymmetric deformations, may be expressed in the form

$\mathbf{u}=u(R, Z) \mathbf{e}_{r}+w(R, Z) \mathbf{e}_{z}$

The deformation gradient tensor $\mathbf{F}=$ Grad $\mathbf{x}$ may be calculated by using

Grad $=\mathbf{E}_{R} \frac{\partial}{\partial R}+\mathbf{E}_{\Theta} \frac{1}{R} \frac{\partial}{\partial \Theta}+\mathbf{E}_{Z} \frac{\partial}{\partial Z}$,

which yields

$\mathbf{F}=\left(1+u_{R}\right) \mathbf{e}_{r} \otimes \mathbf{E}_{R}+u_{Z} \mathbf{e}_{r} \otimes \mathbf{E}_{Z}+\left(1+\frac{u}{R}\right) \mathbf{e}_{\theta} \otimes \mathbf{E}_{\Theta}+w_{R} \mathbf{e}_{z} \otimes \mathbf{E}_{R}$

$+\left(1+w_{Z}\right) \mathbf{e}_{Z} \otimes \mathbf{E}_{Z}$

where the subscripts $R$ and $Z$ on $u$ and $w$ indicate the partial derivatives $\partial / \partial R$ and $\partial / \partial Z$, respectively. The matrix representation of (5) with respect to both sets of cylindrical polar coordinates is

$\mathrm{F}=\left(\begin{array}{ccc}1+u_{R} & 0 & u_{Z} \\ 0 & 1+u / R & 0 \\ w_{R} & 0 & 1+w_{Z}\end{array}\right)$

Using (5), we may calculate the right Cauchy-Green deformation tensor, defined by $\mathbf{C}=\mathbf{F}^{\mathrm{T}} \mathbf{F}$, where ${ }^{\mathrm{T}}$ denotes the transpose. This yields

$$
\begin{aligned}
\mathbf{C}= & {\left[\left(1+u_{R}\right)^{2}+w_{R}^{2}\right] \mathbf{E}_{R} \otimes \mathbf{E}_{R}+(1+u / R)^{2} \mathbf{E}_{\Theta} \otimes \mathbf{E}_{\Theta} } \\
& +\left[u_{Z}^{2}+\left(1+w_{Z}\right)^{2}\right] \mathbf{E}_{Z} \otimes \mathbf{E}_{Z}+\left[u_{Z}\left(1+u_{R}\right)+\left(1+w_{Z}\right) w_{R}\right] \\
& \times\left(\mathbf{E}_{R} \otimes \mathbf{E}_{Z}+\mathbf{E}_{Z} \otimes \mathbf{E}_{R}\right) .
\end{aligned}
$$

We also note the polar decomposition $\mathbf{F}=\mathbf{R U}$, where $\mathbf{R}$ is a proper orthogonal tensor and $\mathbf{U}$ is the right stretch tensor, which is positive definite and symmetric. Thus, $\mathbf{C}=\mathbf{U}^{2}$. The eigenvalues of $\mathbf{U}$ are the principal stretches of the deformation, denoted $\lambda_{i}, i=1,2$, 3 . The principal axes of $\mathbf{C}$ and $\mathbf{U}$ coincide and we can see immediately from (6) that $\mathbf{E}_{\Theta}$ is a (Lagrangian) principal axis, which corresponds to the principal stretch $\lambda_{2}=1+u / R$. The other two principal axes lie parallel to the $(R, Z)$ plane and can be defined in terms of an angle $\psi$ via

$\mathbf{E}_{R}^{\prime}=\cos \psi \mathbf{E}_{R}+\sin \psi \mathbf{E}_{Z}, \quad \mathbf{E}_{Z}^{\prime}=-\sin \psi \mathbf{E}_{R}+\cos \psi \mathbf{E}_{Z}$.

The corresponding principal stretches are taken as $\lambda_{1}$ and $\lambda_{3}$, respectively. Then, we have

$\mathbf{C}=\lambda_{1}^{2} \mathbf{E}_{Z}^{\prime} \otimes \mathbf{E}_{R}^{\prime}+\lambda_{2}^{2} \mathbf{E}_{\Theta} \otimes \mathbf{E}_{\Theta}+\lambda_{3}^{2} \mathbf{E}_{Z}^{\prime} \otimes \mathbf{E}_{Z}^{\prime}$ 


\subsection{Material properties and equilibrium}

The material of the tube is considered to be incompressible, so that the constraint

$J=\operatorname{det} \mathbf{F}=\operatorname{det} \mathbf{U}=\lambda_{1} \lambda_{2} \lambda_{3} \equiv 1$

must be satisfied for every material point $\mathbf{X}$. Subject to this constraint, the elastic properties of the material can be described in terms of a strain-energy function $W(\mathbf{F})$, defined per unit volume. By objectivity $W(\mathbf{F})=W(\mathbf{U})$. The associated Biot stress tensor, denoted here by $\mathbf{T}$, is then given by

$\mathbf{T}=\frac{\partial W}{\partial \mathbf{U}}-p \mathbf{U}^{-1}$

where $p$ is a Lagrange multiplier associated with the constraint (9). For details of the Biot stress tensor we refer to Ogden (1997). For the considered deformation $p$ is a function only of $R$ and $Z$.

Now, for an isotropic material $W$ is a function only of the principal stretches $\lambda_{1}, \lambda_{2}, \lambda_{3}$, again subject to (9), and $\mathbf{T}$ has the same principal axes as $\mathbf{U}$. The principal Biot stresses are then simply

$t_{i}=\frac{\partial W}{\partial \lambda_{i}}-p \lambda_{i}^{-1}, \quad i=1,2,3$

Let $\mathbf{S}$ denote the nominal stress tensor. Then, since the material is isotropic, we have

$\mathbf{S}=\mathbf{T R}^{\mathrm{T}}$

where $\mathbf{R}$ is obtained from the polar decomposition as $\mathbf{R}=\mathbf{F} \mathbf{U}^{-1}$. In the absence of body forces the equilibrium equation is expressed in terms of the nominal stress as

$\operatorname{Div} \mathbf{S}=\mathbf{0}$,

where Div is the divergence operator with respect to $\mathbf{X}$. Alternatively, in terms of the Cauchy stress tensor, denoted $\boldsymbol{\sigma}$ and given by $\boldsymbol{\sigma}=\boldsymbol{J}^{-1} \mathbf{F S}$, the equilibrium equation may be written equivalently as

$\operatorname{div} \boldsymbol{\sigma}=\mathbf{0}$.

The principal Cauchy stresses are given by

$\sigma_{i}=\lambda_{i} \frac{\partial W}{\partial \lambda_{i}}-p, \quad i=1,2,3$

On the external lateral surface of the tube a pressure $P$, per unit deformed area, is applied, while the inner surface is kept free of traction. The boundary conditions on these surfaces may then be given as

$\mathbf{S}^{\mathrm{T}} \mathbf{N}= \begin{cases}-P \mathbf{F}^{-\mathrm{T}} \mathbf{N} & \text { on } R=B \\ \mathbf{0} & \text { on } R=A,\end{cases}$

where $\mathbf{N}$ is the unit outward normal to the lateral surface of the tube in the reference configuration, i.e. $\mathbf{N}=\mathbf{E}_{R}$ on $R=B$ and $\mathbf{N}=-\mathbf{E}_{R}$ on $R=A$.

On the ends of the tube the displacement is taken to vanish except for the special case in which we consider the deformation to maintain circular symmetry. Thus,

$u=w=0$ on $Z=0, L$

For the specific calculations we make use of the neo-Hookean strain-energy function, which is given by

$W=\frac{1}{2} \mu\left(\lambda_{1}^{2}+\lambda_{2}^{2}+\lambda_{3}^{2}-3\right)$,

where the constant $\mu$ is the shear modulus of the material.

\section{Linear and nonlinear equations}

We consider the nonlinear formulation with the boundary conditions specified above together with two special cases: the first is nonlinear but assumes that the deformation is radially symmetric, for which an analytical solution is obtained, while the second is based on the linear theory of elasticity. These special cases serve to verify our $\mathrm{C}++$ code and to highlight, in particular, the differences between the linear and nonlinear results.

\subsection{Radially-symmetric case}

If the deformation is radially symmetric then the deformed geometry has the form

$a \leq r \leq b, \quad 0 \leq \theta \leq 2 \pi, \quad 0 \leq z \leq l$,

where $a$ and $b$, respectively, are the deformed inner and outer radii of the tube and $l$ is its length.

For this special case, we assume that the displacement is given by $\mathbf{u}=u(R) \mathbf{e}_{r}$, so that there is no dependence on $Z$ and $w$ is identically zero. Then the deformation gradient tensor $\mathbf{F}$ in (5) specializes accordingly, and the right Cauchy-Green deformation tensor in $(6)$ reduces to

$\mathbf{C}=\left(1+u_{R}\right)^{2} \mathbf{E}_{R} \otimes \mathbf{E}_{R}+(1+u / R)^{2} \mathbf{E}_{\Theta} \otimes \mathbf{E}_{\Theta}+\mathbf{E}_{Z} \otimes \mathbf{E}_{Z}$.

It follows that the Lagrangian principal axes coincide with the basis vectors $\mathbf{E}_{R}, \mathbf{E}_{\Theta}, \mathbf{E}_{Z}$ and the principal stretches are

$\lambda_{1}=1+u_{R}, \quad \lambda_{2}=1+\frac{u}{R}, \quad \lambda_{3}=1$.

Furthermore, $\mathbf{S}=\mathbf{T}$ and hence

$\mathbf{S}=t_{1} \mathbf{E}_{R} \otimes \mathbf{E}_{R}+t_{2} \mathbf{E}_{\Theta} \otimes \mathbf{E}_{\Theta}+t_{3} \mathbf{E}_{Z} \otimes \mathbf{E}_{Z}$

The equilibrium equation (13) specializes to the single component

$S_{R r, R}+\frac{1}{R}\left(S_{R r}-S_{\Theta \theta}\right)=0$,

where $S_{R r}=t_{1}, S_{\Theta \theta}=t_{2}$ and, $R \equiv \mathrm{d} / \mathrm{d} R$. For the neo-Hookean material (18) we then obtain, on use of (21),

$t_{1}=\mu \lambda_{1}-p \lambda_{2}, \quad t_{2}=\mu \lambda_{2}-p \lambda_{1}, \quad t_{3}=\mu-p$,

where the incompressibility condition $\lambda_{1} \lambda_{2}=1$, or equivalently

$u+(R+u) u_{R}=0$,

has been used. The latter can be integrated to give $r=R+u$ in the form

$r^{2}=R^{2}+a^{2}-A^{2}$.

The component form of the boundary condition (16) may now be written

$S_{R r} \equiv t_{1}= \begin{cases}-P \lambda_{2} & \text { on } R=B \\ 0 & \text { on } R=A .\end{cases}$

Using (24) and noting that $R \lambda_{2, R}=\lambda_{1}-\lambda_{2}$ we may integrate (23) and use the boundary conditions (27) to obtain

$\left.P=\mu \ln \left(\frac{A b}{B a}\right)+\frac{1}{2} \mu \frac{A^{2}}{a^{2}}-\frac{B^{2}}{b^{2}}\right)$. 


\subsection{The linear case}

In the linear theory of incompressible isotropic elasticity the (Cauchy) stress tensor is given by

$\boldsymbol{\sigma}=-p \mathbf{I}+\mu\left[\operatorname{grad} \mathbf{u}+(\operatorname{grad} \mathbf{u})^{\mathrm{T}}\right]$,
Recalling that the Biot stress tensor has the same principal axes as $\mathbf{U}$ we may write

$\mathbf{T}=t_{1} \mathbf{E}_{R}^{\prime} \otimes \mathbf{E}_{R}^{\prime}+t_{2} \mathbf{E}_{\Theta}^{\prime} \otimes \mathbf{E}_{\Theta}^{\prime}+t_{3} \mathbf{E}_{Z}^{\prime} \otimes \mathbf{E}_{Z}^{\prime}$,

and hence from (12) with $\mathbf{R}=\mathbf{F U}^{-1}$, we obtain the components of the nominal stress tensor in the form

$$
\begin{aligned}
& S_{R r}=\left(\lambda_{1}^{-1} t_{1}-\lambda_{3}^{-1} t_{3}\right) u_{Z} \sin \psi \cos \psi+\left(1+u_{R}\right)\left(\lambda_{1}^{-1} t_{1} \cos ^{2} \psi+\lambda_{3}^{-1} t_{3} \sin ^{2} \psi\right), \\
& S_{R z}=\left(\lambda_{1}^{-1} t_{1}-\lambda_{3}^{-1} t_{3}\right)\left(1+w_{Z}\right) \sin \psi \cos \psi+w_{R}\left(\lambda_{1}^{-1} t_{1} \cos ^{2} \psi+\lambda_{3}^{-1} t_{3} \sin ^{2} \psi\right), \\
& S_{Z r}=\left(\lambda_{1}^{-1} t_{1}-\lambda_{3}^{-1} t_{3}\right)\left(1+u_{R}\right) \sin \psi \cos \psi+u_{Z}\left(\lambda_{1}^{-1} t_{1} \sin ^{2} \psi+\lambda_{3}^{-1} t_{3} \cos ^{2} \psi\right), \\
& S_{Z z}=\left(\lambda_{1}^{-1} t_{1}-\lambda_{3}^{-1} t_{3}\right) w_{R} \sin \psi \cos \psi+\left(1+w_{z}\right)\left(\lambda_{1}^{-1} t_{1} \sin ^{2} \psi+\lambda_{3}^{-1} t_{3} \cos ^{2} \psi\right),
\end{aligned}
$$

where $\mathbf{I}$ is the identity tensor.

Then, for the axisymmetric situation, the equilibrium equation (14) has two components that are not satisfied trivially, namely

$\sigma_{r r, r}+\sigma_{z r, z}+\frac{1}{r}\left(\sigma_{r r}-\sigma_{\theta \theta}\right)=0$,

$\sigma_{r z, r}+\sigma_{z z, z}+\frac{1}{r} \sigma_{r z}=0$,

and the incompressibility constraint is

$u_{r}+\frac{u}{r}+w_{z}=0$.

The boundary conditions (17) are unchanged, but (16) may be reduced to

$\boldsymbol{\sigma n}= \begin{cases}-P \mathbf{n} & \text { on } R=B \\ \mathbf{0} & \text { on } R=A,\end{cases}$

there being no distinction between the deformed and reference configurations.

\subsection{The nonlinear case}

Comparing (6) and (8) and using (7), we obtain

$\lambda_{1}^{2} \cos ^{2} \psi+\lambda_{3}^{2} \sin ^{2} \psi=\left(1+u_{R}\right)^{2}+w_{R}^{2}$,

$\lambda_{1}^{2} \sin ^{2} \psi+\lambda_{3}^{2} \cos ^{2} \psi=u_{Z}^{2}+\left(1+w_{Z}\right)^{2}$,

$\left(\lambda_{1}^{2}-\lambda_{3}^{2}\right) \sin \psi \cos \psi=u_{Z}\left(1+u_{R}\right)+w_{R}\left(1+w_{Z}\right)$,

and $\lambda_{2}=1+u / R$. From (34) to (36), it follows that

$\left(\lambda_{1}^{2}-\lambda_{3}^{2}\right) \cos 2 \psi=w_{R}^{2}-u_{Z}^{2}+\left(1+u_{R}\right)^{2}-\left(1+w_{Z}\right)^{2}$,

$\left(\lambda_{1}^{2}-\lambda_{3}^{2}\right) \sin 2 \psi=2\left[u_{Z}\left(1+u_{R}\right)+w_{R}\left(1+w_{Z}\right)\right]$.

It turns out that we must take $\lambda_{1}>\lambda_{3}$, and hence we obtain

$$
\begin{aligned}
2 \lambda_{1}= & \sqrt{\left(u_{Z}-w_{R}\right)^{2}+\left(u_{R}+w_{Z}+2\right)^{2}} \\
& +\sqrt{\left(u_{Z}+w_{R}\right)^{2}+\left(u_{R}-w_{Z}\right)^{2}}, \\
2 \lambda_{3}= & \sqrt{\left(u_{Z}-w_{R}\right)^{2}+\left(u_{R}+w_{Z}+2\right)^{2}} \\
& -\sqrt{\left(u_{Z}+w_{R}\right)^{2}+\left(u_{R}-w_{Z}\right)^{2}} .
\end{aligned}
$$

together with $S_{\Theta \theta}=t_{2}$.

The appropriate specialization of the equilibrium equation (13) then yields the two equations

$S_{R r, R}+S_{Z r, Z}+\frac{1}{R}\left(S_{R r}-S_{\Theta \theta}\right)=0$,

$S_{R z, R}+S_{Z z, Z}+\frac{1}{R} S_{R z}=0$,

the incompressible condition is

$(1+u / R)\left[\left(1+u_{R}\right)\left(1+w_{Z}\right)-u_{Z} w_{R}\right]=1$,

and the boundary condition (16) specializes to

$S_{R r}= \begin{cases}-P(1+u / R)\left(1+w_{Z}\right) & \text { on } R=B \\ 0 & \text { on } R=A,\end{cases}$

with

$S_{R z}= \begin{cases}P(1+u / R) u_{Z} & \text { on } R=B \\ 0 & \text { on } R=A .\end{cases}$

\section{Finite element algorithm}

To solve the nonlinear partial differential equations, the objectoriented package Libmesh (Kirk et al., 2006) is used, which is a framework for solving and analyzing systems of nonlinear equations using the finite element method. It is also an interface to the high quality software PETSc, which is used to solve linear systems on both serial and parallel platforms.

\subsection{Discretization}

We discretize the governing PDEs (13) with the constraint (9) using the weighted residual-Galerkin method. The elastic domain is divided into a set of sub-domains. Libmesh offers the options of quadratic elements of 9-node quadrilateral and 6-node triangle type. Using a mixed interpolation approach, the displacement components $u, w$ and the radial coordinate $R$ are interpolated by quadratic shape functions $N_{i}$, while the Lagrange multiplier $p$ is interpolated by linear shape functions $L_{i}$, i.e.

$$
\begin{array}{ll}
u=\sum_{k=1}^{n_{1}} N_{k}(\xi, \eta) u_{k}, & w=\sum_{k=1}^{n_{1}} N_{k}(\xi, \eta) w_{k}, \\
R=\sum_{k=1}^{n_{1}} N_{k}(\xi, \eta) R_{k}, & p=\sum_{k=1}^{n_{2}} L_{k}(\xi, \eta) p_{k},
\end{array}
$$

where $n_{1}, n_{2}$ are the element node numbers, which are dependent on the element type chosen, and $\xi$ and $\eta$ are natural coordinate variables, corresponding to isoparametric finite elements. 
This allows us to write the discretized nonlinear governing equations as

$\mathfrak{R}=\mathbf{K}(\mathbf{U}) \mathbf{U}-\mathbf{F}(\mathbf{U})=\mathbf{0}$,

where $\mathbf{U}$ is the global vector of unknowns, $\mathbf{K}(\mathbf{U})$ is the global stiffness matrix, $\mathbf{F}(\mathbf{U})$ denotes the force vector, which is also dependent on $\mathbf{U}$, and $\mathfrak{R}$ is the global residual vector, which should be $\mathbf{0}$ for an exact solution. Note that $\mathbf{U}$ was used earlier for the right stretch tensor, which does not appear hereon so there is no conflict of notation. Numerical simulations show that the 6-node triangle is more efficient than the 9-node quadrilateral element for large distortions. The formulation of the finite element matrices is problem dependent, as shown in Section 4.3 below.

\subsection{Newton's method}

To solve systems of nonlinear equations such as (48), the SNES library of PETSc (Balay et al., 2008) is called by Libmesh. The SNES library provides a powerful suite of numerical routines, and Newton-Krylov methods provide the core of the package, including line search and trust region techniques. Newton's iteration may be implemented by

$\mathbf{U}_{r+1}=\mathbf{U}_{r}-\mathbf{J}^{-1}\left(\mathbf{U}_{r}\right) \mathfrak{R}\left(\mathbf{U}_{r}\right)$,

where $r$ is the iteration number and $\mathbf{J}$ is the Jacobian matrix, which, by using (48), is defined by

$\mathbf{J}\left(\mathbf{U}_{r}\right)=\frac{\partial \Re\left(\mathbf{U}_{r}\right)}{\partial \mathbf{U}}=\mathbf{K}\left(\mathbf{U}_{r}\right)+\frac{\partial \mathbf{K}\left(\mathbf{U}_{r}\right)}{\partial \mathbf{U}} \mathbf{U}_{r}-\frac{\partial \mathbf{F}\left(\mathbf{U}_{r}\right)}{\partial \mathbf{U}}$.

Convergence is achieved when the relative residual tolerance $\left\|\mathfrak{R}\left(\mathbf{U}_{r}\right)\right\| /\left\|\mathfrak{R}\left(\mathbf{U}_{0}\right)\right\|$ (in the $l 2$ norm) is less than $10^{-8}$ or the absolute tolerance $\left\|\mathfrak{R}\left(\mathbf{U}_{r}\right)\right\|$ is less than $10^{-12}$, where $\left\|\mathfrak{R}\left(\mathbf{U}_{0}\right)\right\|$ is the initial residual.

\subsection{Detailed discretizing integrations}

\subsubsection{Radially-symmetric case}

Applying Galerkin's method to Eq. (23), we obtain

$\int_{\Omega} N_{i} S_{R r, R} \mathrm{~d} \Omega+\int_{\Omega} N_{i} \frac{1}{R}\left(S_{R r}-S_{\Theta \theta}\right) \mathrm{d} \Omega=0$,

where $\Omega$ is the integration domain. The domain of integration $\Omega$ is the physical domain in the reference configuration corresponding to the $(R, Z)$ tube section. For each element, (51) can be integrated by parts to give
Equation (25) may be discretized similarly to give

$\sum_{j=1}^{n_{1}} \int_{R} \int_{Z} R L_{i}\left[N_{j}+\left(R+\sum_{k=1}^{n_{1}} N_{k} u_{k}\right) N_{j, R}\right] \mathrm{d} R \mathrm{~d} Z u_{j}=0$.

Here we have adopted 9-node quadrilateral elements in order to achieve better accuracy, so that $n_{1}=9, n_{2}=4$. Note that when assembled globally the boundary integrals in (52) cancel out except at the boundaries of the tube.

\subsubsection{The linear case}

The discretized equations for the linear case can be obtained by using a similar procedure to that used for the radially-symmetric case. This yields

$$
\begin{aligned}
& \sum_{j=1}^{n_{1}} \int_{r} \int_{z}\left(\mu r\left(2 N_{i, r} N_{j, r}+N_{i, z} N_{j, z}\right)+2 \frac{\mu}{r} N_{i} N_{j}\right) \mathrm{d} r \mathrm{~d} z u_{j} \\
& \quad+\sum_{j=1}^{n_{1}} \int_{r} \int_{z} \mu r N_{i, z} N_{j, r} \mathrm{~d} r \mathrm{~d} z w_{j}-\sum_{j=1}^{n_{2}} \int_{r} \int_{z}\left(r N_{i, r}+N_{i}\right) L_{j} \mathrm{~d} r \mathrm{~d} z p_{j} \\
& \quad=\left.\int_{Z}\left(r N_{i} \sigma_{r r}\right)\right|_{r_{1}} ^{r_{2}} \mathrm{~d} z+\left.\int_{r} r\left(N_{i} \sigma_{z r}\right)\right|_{z_{1}} ^{z_{2}} \mathrm{~d} r
\end{aligned}
$$

$$
\begin{aligned}
& \sum_{j=1}^{n_{1}} \int_{r} \int_{z} \mu r N_{i, r} N_{j, z} \mathrm{~d} r \mathrm{~d} z u_{j}+\sum_{j=1}^{n_{1}} \int_{r} \int_{z} \mu r\left(N_{i, r} N_{j, r}\right. \\
& \left.+2 N_{i, z} N_{j, z}\right) \mathrm{d} r \mathrm{~d} z w_{j}-\sum_{j=1}^{n_{2}} \int_{r} \int_{z} r N_{i, z} L_{j} \mathrm{~d} r \mathrm{~d} z p_{j} \\
& =\left.\int_{z}\left(r N_{i} \sigma_{r z}\right)\right|_{r_{1}} ^{r_{2}} \mathrm{~d} z+\left.\int_{r} r\left(N_{i} \sigma_{z z}\right)\right|_{z_{1}} ^{z_{2}} \mathrm{~d} r
\end{aligned}
$$

$$
\begin{aligned}
& \sum_{j=1}^{n_{1}} \int_{r} \int_{Z} r L_{i}\left(N_{j, r}+\frac{1}{r} N_{j}\right) \mathrm{d} r \mathrm{~d} z u_{j}+\sum_{j=1}^{n_{1}} \int_{r} \int_{Z} r L_{i} N_{j, z} \mathrm{~d} r \mathrm{~d} z w_{j} \\
& =0 .
\end{aligned}
$$

For the linear case, the 6-node triangle is used, so that $n_{1}=6, n_{2}=3$. This is also used for the following nonlinear case since for large distortions the triangular element shows its superiority over the rectangular element.

$$
\begin{aligned}
& -\sum_{j=1}^{n_{1}} \int_{R} \int_{Z}\left(\frac{1}{R} N_{i} N_{j}+R N_{i, R} N_{j, R}\right) \mathrm{d} R \mathrm{~d} Z u_{j} \\
& \left.+\sum_{j=1}^{n_{2}} \int_{R} \int_{Z} L_{j}\left[\left(1+\sum_{k=1}^{n_{1}} N_{k, R} u_{k}\right) N_{i}+R+\sum_{k=1}^{n_{1}} N_{k} u_{k}\right) N_{i, R}\right] \mathrm{d} R \mathrm{~d} Z p_{j} \\
& =-\left.\int_{Z}\left(R N_{i} S_{R r}\right)\right|_{R_{1}} ^{R_{2}} \mathrm{~d} Z+\int_{R} \int_{Z}\left(N_{i}+R N_{i, R}\right) \mathrm{d} R \mathrm{~d} Z .
\end{aligned}
$$




$$
\begin{aligned}
& \sum_{j=1}^{n_{1}} \int_{R} \int_{Z} \frac{\mu}{R} N_{i} N_{j}+R N_{i, R}\left\{\left[\frac{(1+\cos 2 \psi) t_{1}}{2 \lambda_{1}}+\frac{(1-\cos 2 \psi) t_{3}}{2 \lambda_{3}}\right] N_{j, R}+\frac{1}{2} \sin 2 \psi\left(\frac{t_{1}}{\lambda_{1}}-\frac{t_{3}}{\lambda_{3}}\right) N_{j, Z}\right\} \\
& +R N_{i, Z}\left\{\frac{1}{2} \sin 2 \psi\left(\frac{t_{1}}{\lambda_{1}}-\frac{t_{3}}{\lambda_{3}}\right) N_{j, R}+\left[\frac{(1-\cos 2 \psi) t_{1}}{2 \lambda_{1}}+\frac{(1+\cos 2 \psi) t_{3}}{2 \lambda_{3}}\right] N_{j, Z}\right\} d R d Z u_{j} \\
& -\sum_{j=1}^{n_{2}} \int_{R} \int_{Z} R\left[\frac{1}{R+u} N_{i}+\frac{1+\cos 2 \psi}{2} \lambda_{1}^{-2} N_{i, R}\right] L_{j} \mathrm{~d} R \mathrm{~d} Z p_{j}=-\iint_{R} \mu N_{i} \mathrm{~d} R \mathrm{~d} Z \\
& -\int_{R} \int_{Z} R N_{i, R}\left[\frac{1+\cos 2 \psi}{2} \mu+\frac{(1-\cos 2 \psi) t_{3}}{2 \lambda_{3}}\right] \mathrm{d} R \mathrm{~d} Z-\int_{R} \int_{Z} \frac{1}{2} R N_{i, Z} \sin 2 \psi\left(\frac{t_{1}}{\lambda_{1}}-\frac{t_{3}}{\lambda_{3}}\right) \mathrm{d} R \mathrm{~d} Z \\
& +\left.\int_{Z}\left(R N_{i} S_{R r}\right)\right|_{R_{1}} ^{R_{2}} \mathrm{~d} Z+\left.\int_{R} R\left(N_{i} S_{Z r}\right)\right|_{Z_{1}} ^{Z_{2}} \mathrm{~d} R,
\end{aligned}
$$

$$
\begin{aligned}
& \sum_{j=1}^{n_{1}} \int_{R} \int_{Z} R N_{i, R}\left\{\left[\frac{(1+\cos 2 \psi) t_{1}}{2 \lambda_{1}}+\frac{(1-\cos 2 \psi) t_{3}}{2 \lambda_{3}}\right] N_{j, R}+\frac{1}{2} \sin 2 \psi\left(\frac{t_{1}}{\lambda_{1}}-\frac{t_{3}}{\lambda_{3}}\right) N_{j, Z}\right\} \\
& +R N_{i, Z}\left\{\frac{1}{2} \sin 2 \psi\left(\frac{t_{1}}{\lambda_{1}}-\frac{t_{3}}{\lambda_{3}}\right) N_{j, R}+\left[\frac{(1-\cos 2 \psi) t_{1}}{2 \lambda_{1}}+\frac{(1+\cos 2 \psi) t_{3}}{2 \lambda_{3}}\right] N_{j, Z}\right\} \mathrm{d} R \mathrm{~d} Z w_{j} \\
& -\sum_{j=1}^{n_{2}} \int_{R} \int_{Z} R \frac{1+\cos 2 \psi}{2} \lambda_{3}^{-2} N_{i, Z} L_{j} \mathrm{~d} R \mathrm{~d} Z p_{j}=-\int_{R} \int_{Z} R N_{i, R} \frac{1}{2} \sin 2 \psi\left(\frac{t_{1}}{\lambda_{1}}-\frac{t_{3}}{\lambda_{3}}\right) \mathrm{d} R \mathrm{~d} Z \\
& -\int_{R} \int_{Z} R N_{i, Z}\left[\frac{(1-\cos 2 \psi) t_{1}}{2 \lambda_{1}}+\mu \frac{1+\cos 2 \psi}{2}\right] \mathrm{d} R \mathrm{~d} Z+\left.\int_{Z}\left(R N_{i} S_{R Z}\right)\right|_{R_{1}} ^{R_{2}} \mathrm{~d} Z+\left.\int_{R} R\left(N_{i} S_{Z Z}\right)\right|_{Z_{1}} ^{Z_{2}} \mathrm{~d} R,
\end{aligned}
$$

\subsubsection{The nonlinear case}

On applying Galerkin's method to Eqs. (43)-(45) and integrating by parts, we obtain the stiffness matrix, which can be written in many different ways since the dependent variables are nonlinearly coupled in each of the terms of the stiffness matrix. In general, in the nonlinear case we obtain the discretization by separating off the terms that also appear in the radially-symmetric and linear cases so that the final equations in the nonlinear case can be taken as the corresponding linear ones multiplied by some complicated higher order coefficients. The final forms of the discretized equilibrium equations and the incompressibility condition are

$$
\begin{aligned}
& \sum_{j=1}^{n_{1}} \int_{R} \int_{Z} R L_{i}\left(\frac{1}{R+u} N_{j}+N_{j, R}\right) \mathrm{d} R \mathrm{~d} Z u_{j} \\
& +\sum_{j=1}^{n_{1}} \int_{R} \int_{Z} R L_{i}\left[\left(1+u_{R}\right) N_{j, Z}-u_{Z} N_{j, R}\right] \mathrm{d} R \mathrm{~d} Z w_{j}=0 .
\end{aligned}
$$

Using Eqs. (57)-(59), we obtain the stiffness matrix K. It is worth mentioning that in order to achieve convergence of the solutions $\mathbf{J}$ needs to be computed analytically from (50). Although a much simpler approach to estimating the true Jacobian matrix $\mathbf{J}$ is commonly used by using the stiffness matrix $\mathbf{K}$ this does not work for our nonlinear model. This indicates that the second and third terms in the expression (50) are important and cannot be neglected.

\section{Numerical results}

To demonstrate the differences between the nonlinear and linear cases, three options will be considered for the tube geometry: thick-walled short tubes with $A / B=0.5$ and $L / B=1$, thickwalled longer tubes with $A / B=0.5$ and $L / B=5$, and thin-walled longer tubes with $A / B=0.8$ and $L / B=5$.

Henceforth, all the variables are used in dimensionless form, but without change of notation. The radial coordinates $R$ and $r$ and the displacement components $u$ and $w$ are non-dimensionalized with $B$; the axial coordinates $Z$ and $z$ with $L$; the pressure $P$ and the stress components $\sigma_{i j}$ with the shear modulus $\mu$.

\subsection{Thick-walled short tubes: $A / B=0.5$ and $L / B=1$}

\subsubsection{Displacements and stretches}

As both the linear and nonlinear models should agree when the deformation is small, to validate the numerical approach, a comparison of the nonlinear and linear models for small pressure $(P=0.05)$ has been made, as shown in Fig. 1, in which contour plots of the values of $u$ and $w$ for each case are illustrated, superimposed on the deformed $(r, z)$ section $^{1}$ of the tube. As expected, the distributions of the displacement components $u$ and $w$ for these two cases are virtually indistinguishable. However, the difference between the nonlinear and linear models increases as the pressure increases. This is highlighted in Fig. 2(a), where the displacement $u$ in the radial direction versus the external loading $P$ at point $(R, Z)=(0.5,0.5)$ is shown.

Fig. 2(a) shows that the linear theory overestimates the displacement $u$, especially for large external pressure $(P \gtrsim 1.5)$. For example, for $P=2$, the predictions of $u$ for the linear and nonlinear cases are 0.416 and 0.291 , respectively, an overestimate of $43 \%$. Further validation of our numerical code can be made by comparing the analytical and numerical solutions for the radially-symmetric case shown in Fig. 2(a). The curves are indistinguishable in this figure. Note that the radially-symmetric and nonlinear curves intersect at $P \approx 1.15$. For $P \gtrsim 1.15, u$ increases with $P$ more slowly for the radially-symmetric case than for the nonlinear one, thus significantly underestimating the displacement in the radial direction. The linear theory predicts a smaller axial displacement $w$ than the nonlinear theory, while for 

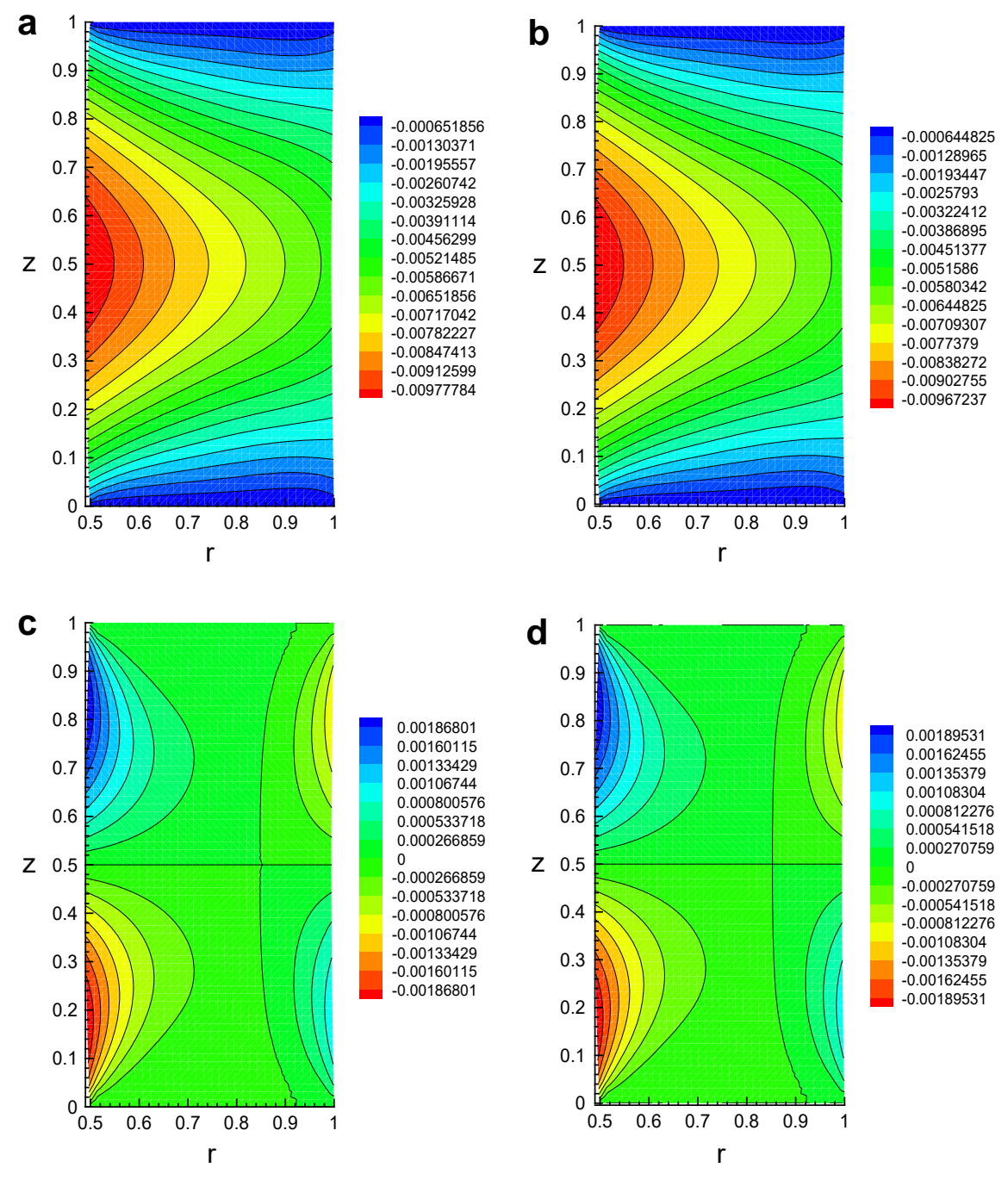

Fig. 1. Deformed profiles of the tube section in $(r, z)$ space for a tube with $A / B=0.5, L / B=1$ subject to external pressure $P=0.05$ with the displacement distributions $u$ and $w$ superimposed: (a) linear $u$; (b) nonlinear $u$; (c) linear $w$; (d) nonlinear $w$. The plots correspond to $R \in[0.5,1], Z \in[0,1]$.

the radially-symmetric case $w=0$; see Fig. 2 (b). The differences in the results for the considered point are representative of those seen at other points, details for which are not shown here.

The deformation, as distinct from the displacement, can be characterized in terms of the stretches, and this is illustrated in

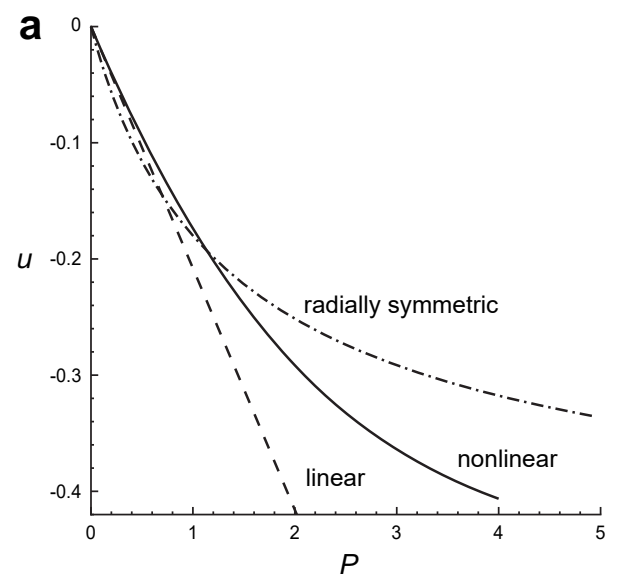

Fig. 3, which shows how the principal stretches at the point $R=0.5$, $Z=0.5$ vary with the pressure $P$. It can be seen that at this point $\lambda_{1}>\lambda_{3}>1$ and $\lambda_{2}<1$. Again, for smaller pressure $(P \lesssim 0.4)$, the principal stretches are almost the same for the linear and nonlinear cases. It is clear, and of course not surprising, that the linear theory

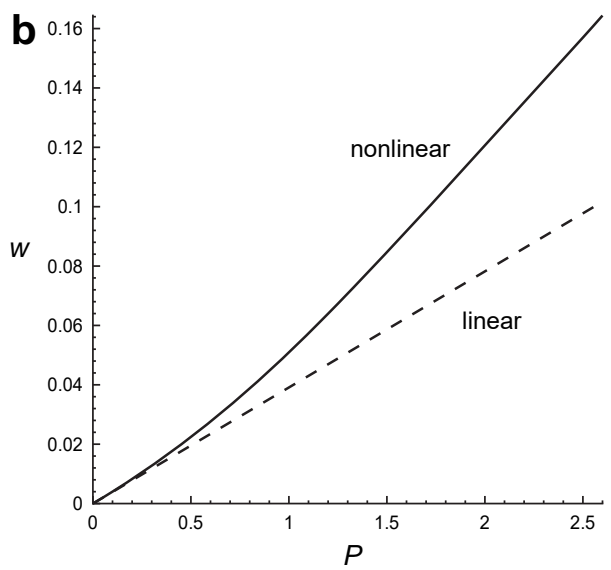

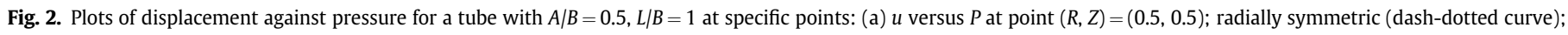
linear (dashed line); nonlinear (solid curve): (b) $w$ versus $P$ at point $(R, Z)=(0.5,0.75)$; linear (dashed line); nonlinear (solid curve). 


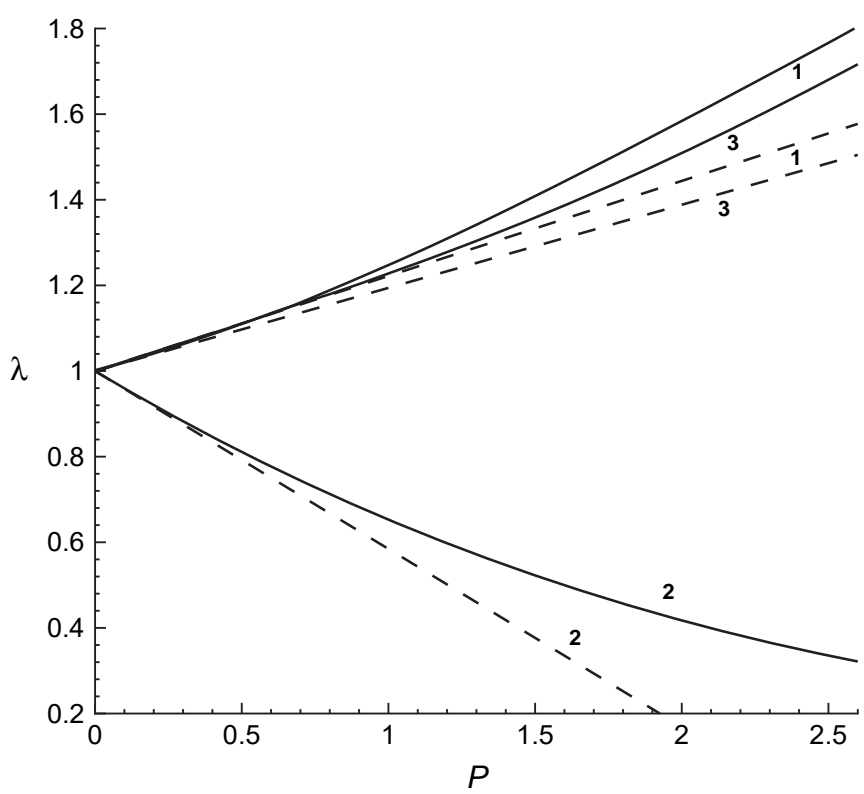

Fig. 3. Plots of $\lambda_{1}, \lambda_{2}$ and $\lambda_{3}$ (labelled $1,2,3$, respectively) versus $P$ calculated at the point $(R, Z)=(0.5,0.5)$ for a tube with $A / B=0.5, L / B=1$ : linear (dashed lines); nonlinear (solid curves).
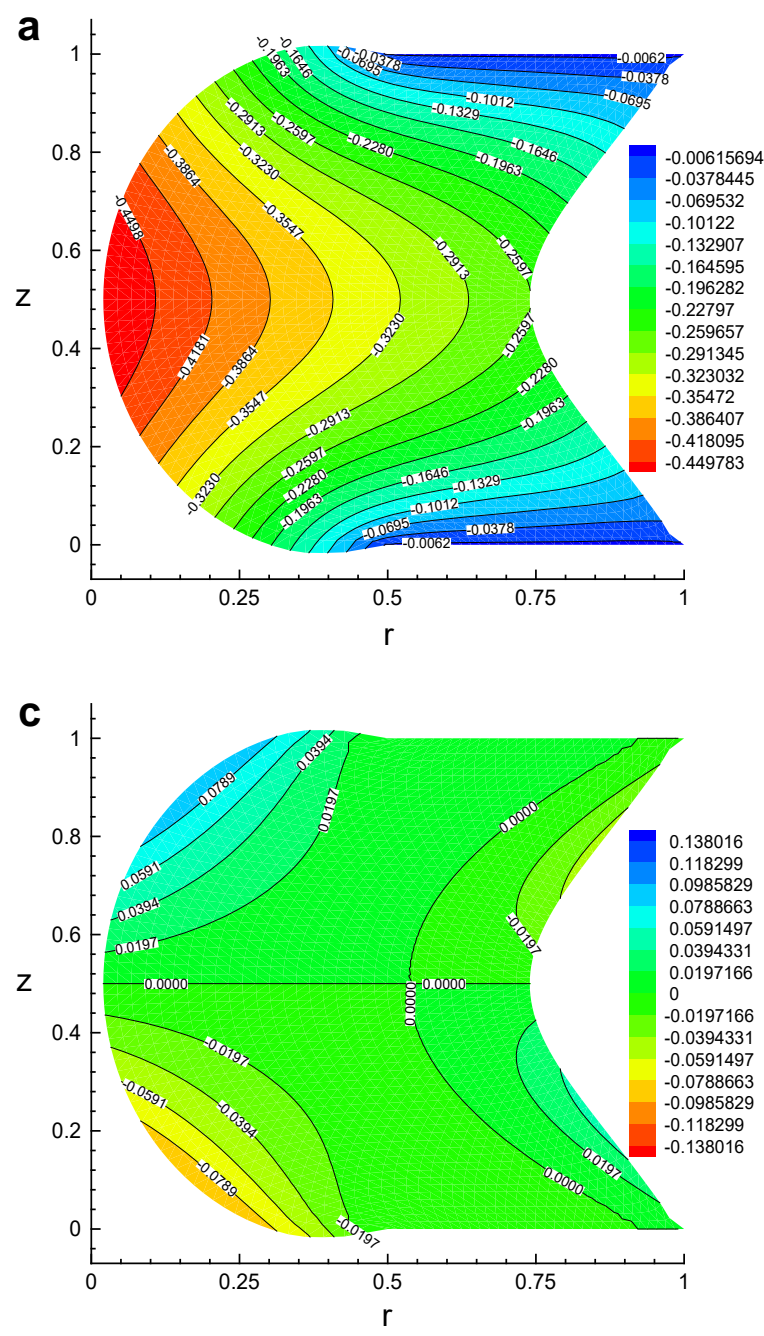

provides an accurate prediction only for small deformations, corresponding to the maximum principal stretch $\lambda_{1}$ less than about 1.1. However, as we shall see in the next section, the linear-nonlinear correspondence reduces to $\lambda_{1}$ less that about 1.05 , i.e. to a strain of about $5 \%$, when the stress components are considered. As the pressure increases the nonlinear theory predicts larger values of $\lambda_{1}$, $\lambda_{2}$ and $\lambda_{3}$ than the linear theory. It should be remarked that the incompressibility condition $\lambda_{1} \lambda_{2} \lambda_{3}=1$ is violated for the principal stretches calculated for the linear theory except for very small values of $P$. This just emphasizes that the linear theory cannot be expected to be valid except for small pressures and the accompanying small deformations.

To better understand the effect of the nonlinear contributions in equations (43)-(45), the displacement distributions are plotted for a relatively large value 2.3 of the pressure $P$ in Fig. 4 . For the purpose of comparison, the corresponding linear results are also shown. Some significant differences between the linear and nonlinear models can be observed in Fig. 4. The displacement in the radial direction is so large in the linear case that the middle section of the tube almost comes into self contact on the axis $R=0$. For the nonlinear case, the most striking feature is the bulging out at the corners, which is barely visible in the linear case. This causes the displacement pattern and magnitude to change. The radial displacement $u$ changes between -0.47 and
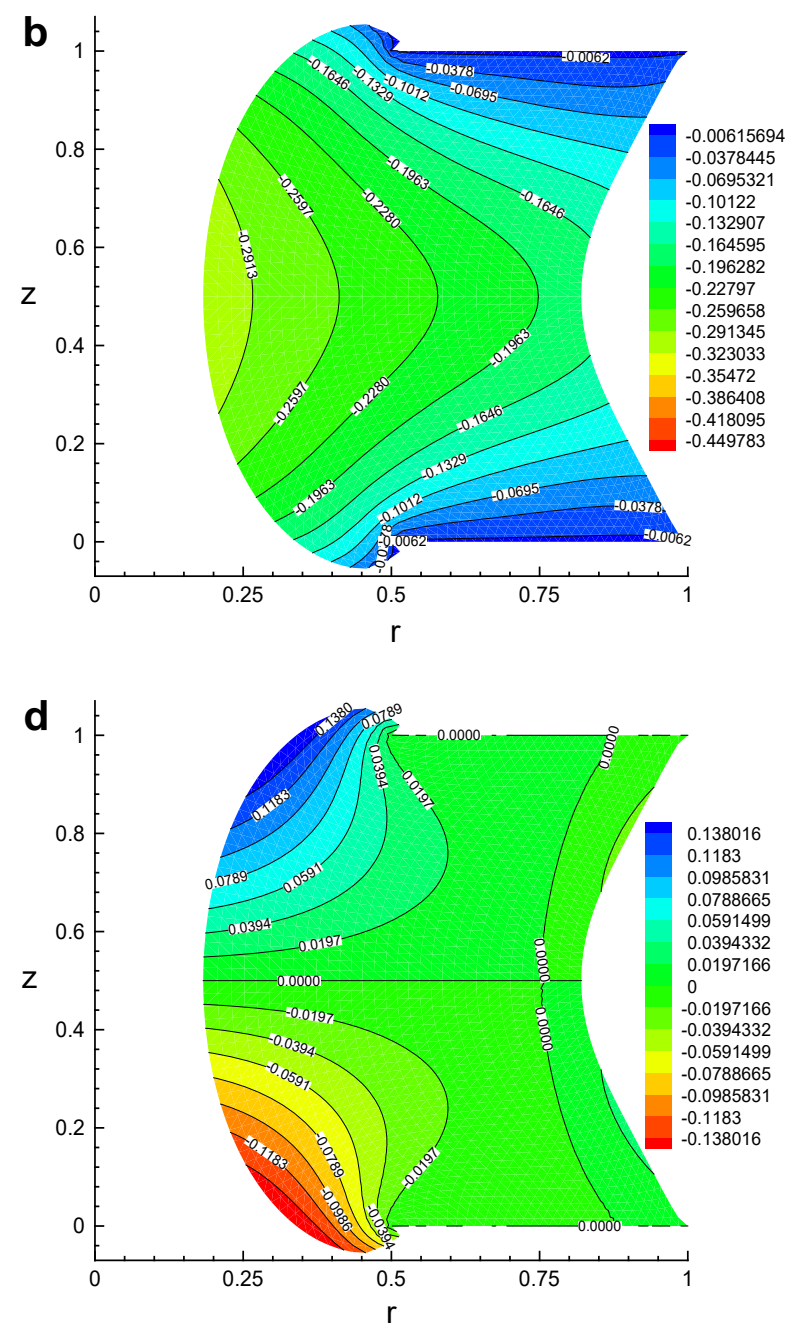

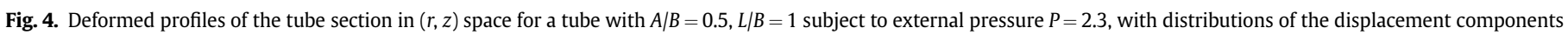
superimposed: (a) linear $u$; (b) nonlinear $u$; (c) linear $w$; (d) nonlinear $w$. 

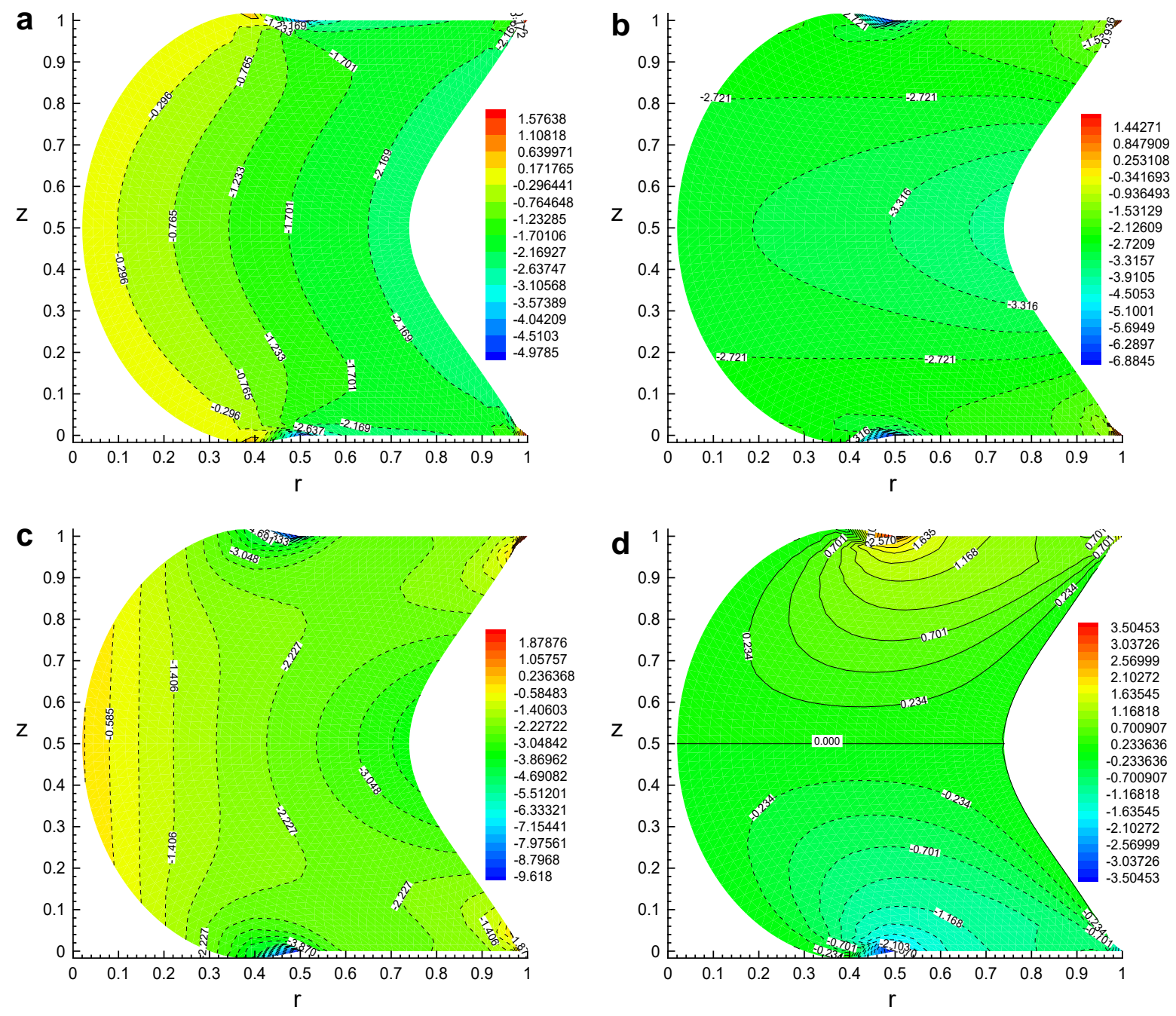

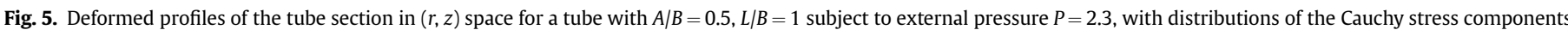
superimposed. Linear case with positive contours (solid), negative contours (dashed): (a) $\sigma_{11}$, (b) $\sigma_{22}$, (c) $\sigma_{33}$, (d) $\sigma_{13}$.

0 in the linear case, and between -0.31 and 0.0145 in nonlinear case. The axial displacement $w$ has the range -0.095 to 0.095 (linear case) and -0.15 to 0.15 (nonlinear case). This is consistent with the corner bulging at $R=0.5$ on the ends of the tube, which stretches the tube section in two opposite axial directions.

\subsubsection{Cauchy stresses}

The stress distributions for the linear and nonlinear cases are again almost the same for very small external pressure, but as expected they depart significantly for large pressures. Figs. 5 and 6 show the distributions of components of Cauchy stress for $P=2.3$ for the linear and nonlinear cases, respectively. Negative values of the stresses are shown with dashed curves. In all cases, the normal stress distributions in the upper half section have mirror symmetry with the lower half, while the distribution of shear stress is antisymmetric.

It can be seen from Figs. 5 and 6 that in both the linear and nonlinear cases, the areas of stress concentration are located at the four corners. However, for the linear case, the normal stresses $\sigma_{11}$, $\sigma_{22}, \sigma_{33}$ are mostly negative, with the peak negative stresses occurring at the two inner corners. The peak positive stresses are at the two outer corners, and in the radial direction (i.e. for $\sigma_{11}$ ). On the inner surface the stress $\sigma_{11}$ is positive only near the ends. This shows that most of the section is under compression when subject to an external pressure. For the nonlinear case, on the other hand, all the peak stresses (positive and negative) occur at the inner corners. This is directly due to the fact that the inner corners are significantly squeezed out, which causes both significant compression and tension there. The shear stress distribution $\sigma_{13}$ is most interesting; for the linear case, $\sigma_{13}$ is entirely positive in the upper half and entirely negative in the lower half, with the zero stress line at $Z=0.5$. However, in the nonlinear case, because of the corner bulging, each half section is divided into four zones between which the shear stress changes sign. In the upper half, the innermost section is sheared upwards, while different parts of the outermost section are subject to either positive or negative shear stress. The opposite is true in the lower half. The general trend for short tubes is that the magnitudes of the stresses in the nonlinear case are smaller than the corresponding linear magnitudes, with $\sigma_{11}$ between -4.7 and $2.11, \sigma_{22}$ between -7.54 and $1.52, \sigma_{33}$ between -8.86 and 2.02 , and $\sigma_{13}$ between -2.87 and 2.87 . These are to be compared with the linear case: $\sigma_{11}$ from -5.44 to $2.04, \sigma_{22}$ from -7.18 to $2.03, \sigma_{33}$ from -10 to 2.69 , and $\sigma_{13}$ from -3.74 to 3.74 .

To show how the stresses change with the external pressure at a particular location, we plot the variation of the stress components $\sigma_{i j}$ with the pressure at point $(R=0.75, Z=0.75)$ in Fig. 7. Again, the differences between the results for the linear and nonlinear models are small if $P$ is small enough, in this case $P \lesssim 0.5$ for the normal 
a
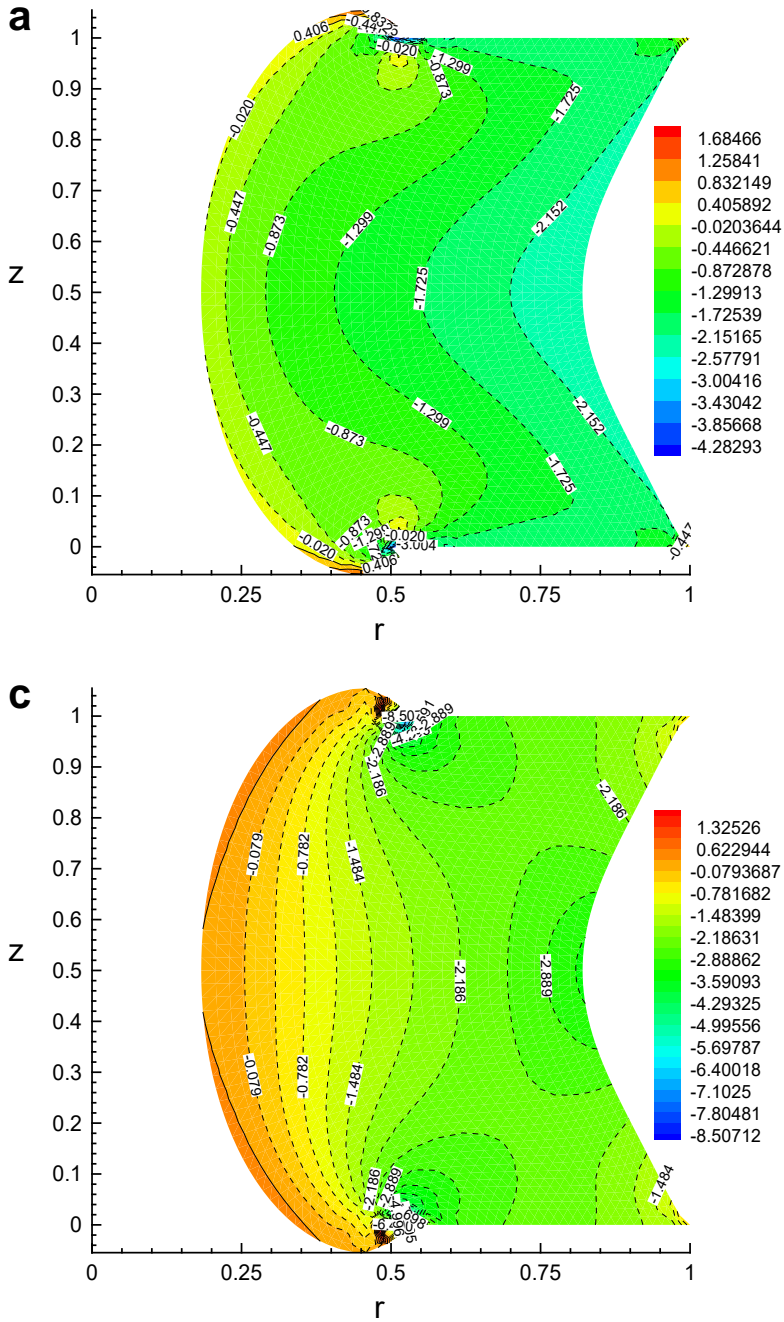

b
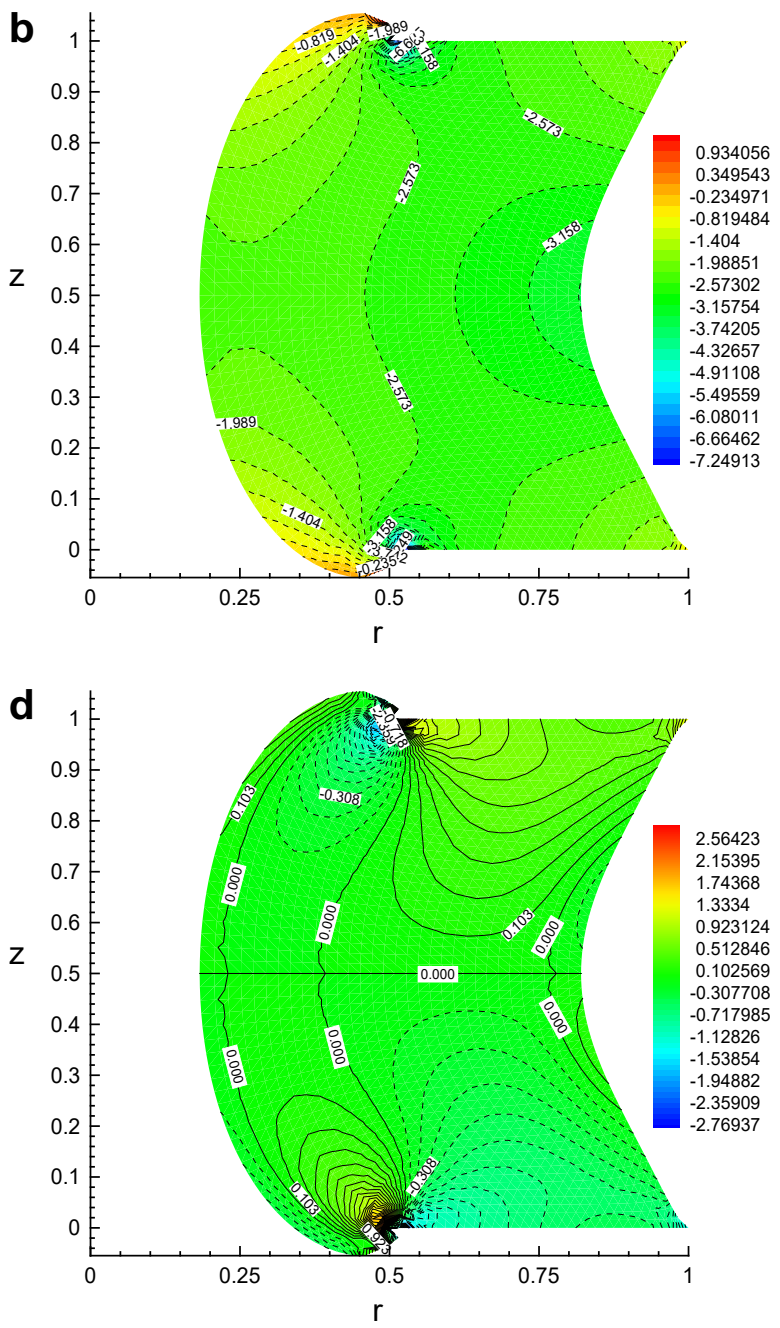

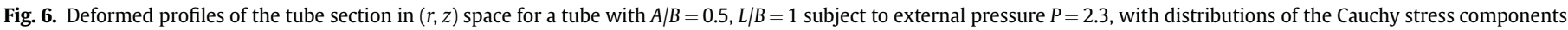
superimposed. Nonlinear case with positive contours (solid), negative contours (dashed): (a) $\sigma_{11}$, (b) $\sigma_{22}$, (c) $\sigma_{33}$, (d) $\sigma_{13}$.

stress components and $P \leqq 0.3$ for $\sigma_{13}$. However, significant differences are found between the linear and nonlinear predictions as $P$ increases, especially in the stress components $\sigma_{11}$ and $\sigma_{13}$. The nonlinear model exhibits much smaller stress magnitudes for the same applied pressure. It is interesting that $\sigma_{13}$ first increases

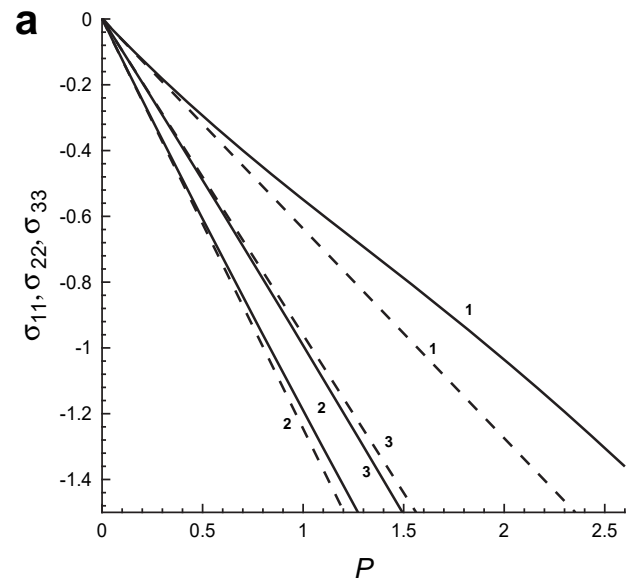

rapidly with $P$, but reaches a maximum around $P=1.8$ and then decreases with further increase in $P$, as shown in Fig. 7(b). This is because as the pressure increases beyond a certain level, the corners bulge out more and more and the second left (negative) shear zone in Fig. 6(d) increases in size, while the third (positive)

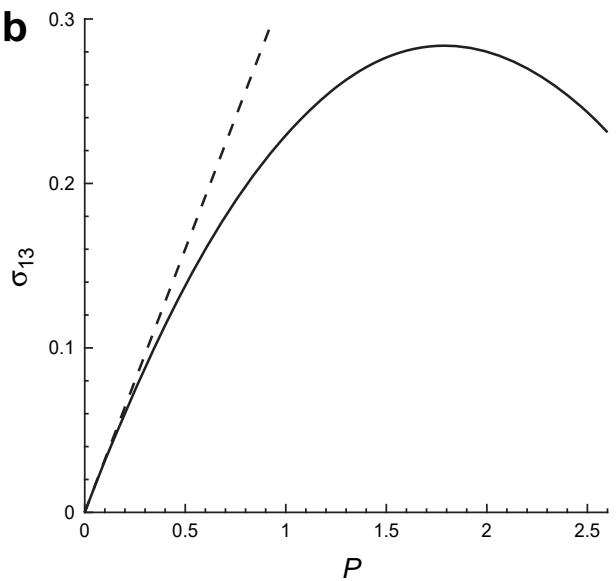

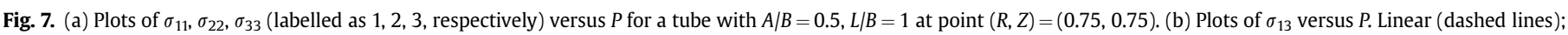
nonlinear (solid curves). 

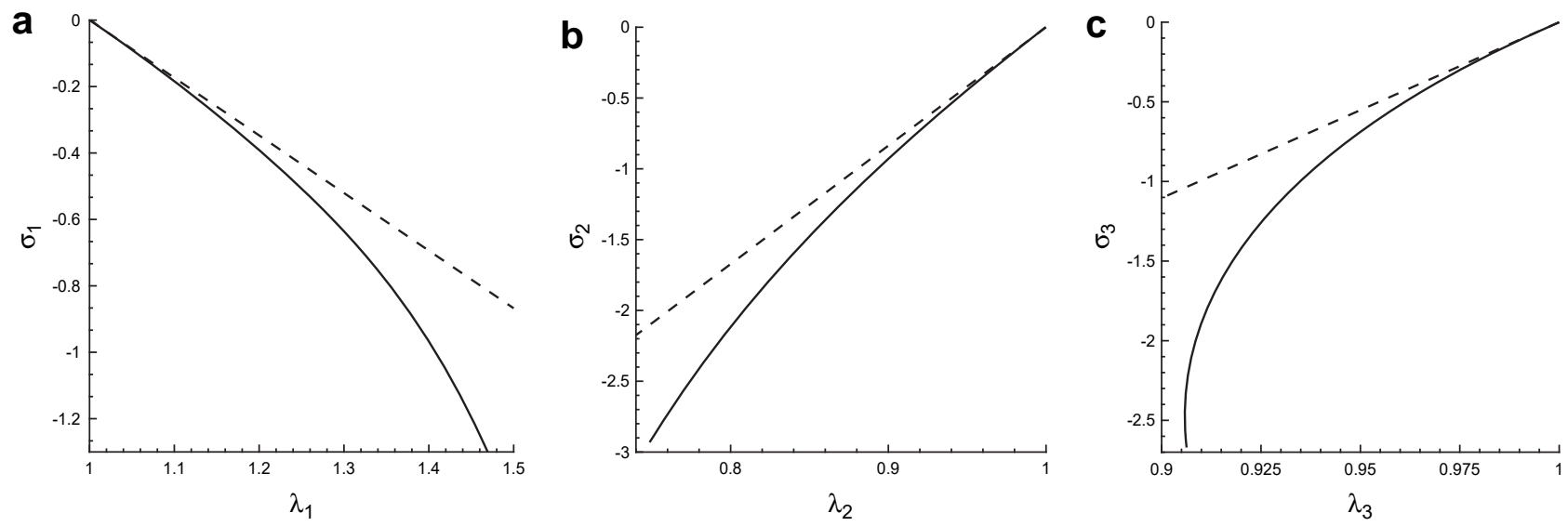

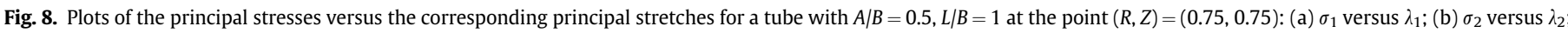
(c) $\sigma_{3}$ versus $\lambda_{3}$. Linear results (dashed lines); nonlinear results (solid curves).

shear zone (where the point is located) shrinks. As a result, the positive shear stress at this point decreases for $P \gtrsim 1.8$.

To illustrate the response of the material locally to the external forces, plots of principal stress versus principal stretch are shown in Fig. 8 for the point $(R=0.75, Z=0.75)$. We note that $\lambda_{1}>1$, $\lambda_{2}<1$ and $\lambda_{3}<1$ at the point in question. Compared with the linear results, the nonlinear model predicts larger magnitudes of the principal stresses for the same principal stretches. This means the stiffness of the material at this special point becomes larger. Note that as $P$ increases the relationship between the principal stress $\sigma_{3}$ and the stretch $\lambda_{3}$ loses monotonicity. It is also noted that at the point $(R=0.75, Z=0.75)$, the angle $\psi$ which defines the principal directions has the constant value $31.6^{\circ}$ for the linear case, while it varies in the range $29.8^{\circ}<\psi<32.6^{\circ}$ for the nonlinear case.

\subsection{Thick-walled longer tubes: $A / B=0.5$ and $L / B=5$}

Next, we consider a tube with the same thickness but five times longer. In this case we find that the $u$ and $w$ versus $P$ curves are similar to those for the shorter tube observed above. The only difference is that for both the linear and nonlinear cases the deformations of longer tubes tend to have two humps instead of one, as suggested in Fig. 9, i.e. the longer tube favours mode- 2 deformations for the range of the pressure applied, while mode- 1 is preferred for the shorter tubes. Fig. 10 shows that the differences in the stress-pressure plots between the linear and nonlinear cases are smaller than for shorter tubes. However, the change in $\sigma_{13}$ for the nonlinear case is interesting. As in Fig. 7(b), it follows the linear curve for small $P$ but the range of values of $P$ for which $\sigma_{13}$ is positive is much smaller in this case, and it bends downwards sharply as soon as $P$ exceeds about 0.1 .

The corresponding principal stress-stretch plots are shown in Fig. 11. The features of Fig. 11(a, b) are similar to those for the shorter tube. However, an interesting change in the $\sigma_{3}-\lambda_{3}$ plot is shown in Fig. 11(c), where an S-shaped curve is observed. This is associated with the complicated pattern of change in the shear zones shown in Fig. 9(a). The nonlinear tube tends to bulge at the two inner corners, which, when combined with the mode- 2 humps, creates a much larger negative shear zone in the upper half of the cylinder. The smaller bulge at the corners also causes the shear stress to be split into negative and positive regions within each half cylinder, and the negative regions emerge and expand as the external pressure increases. The linear case shown in Fig. 9(b) fails to predict the
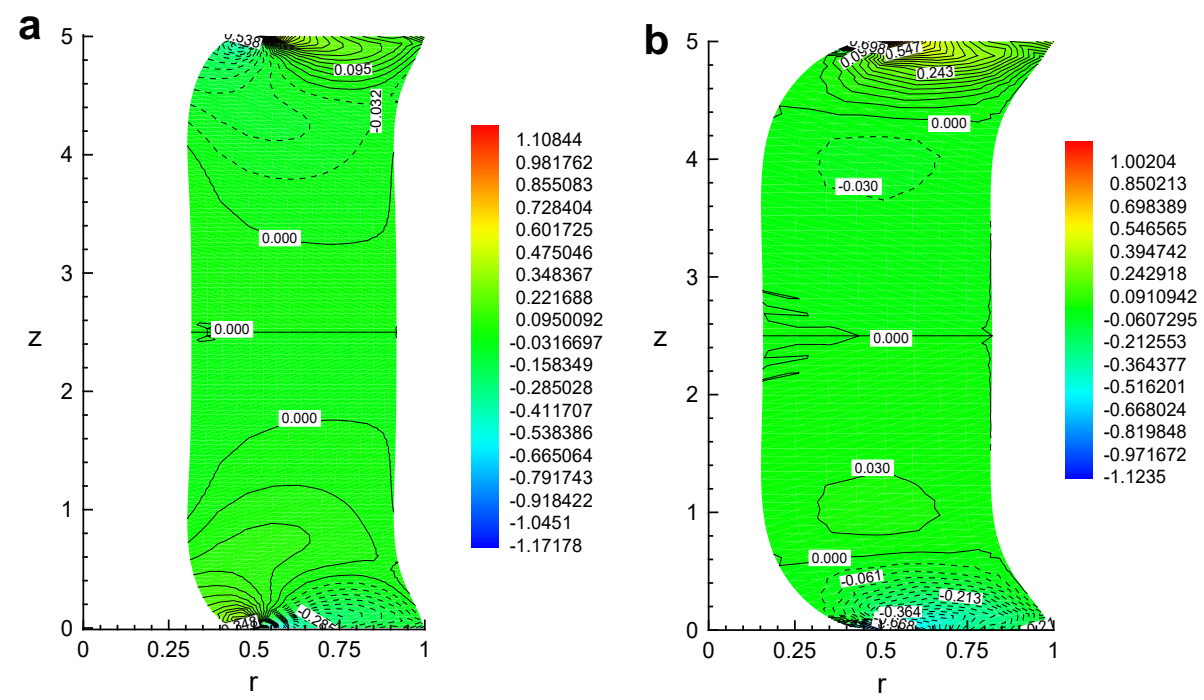

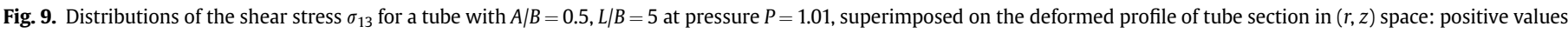
are indicated by solid contours and negative values by dashed contours. (a) nonlinear (b) linear. 

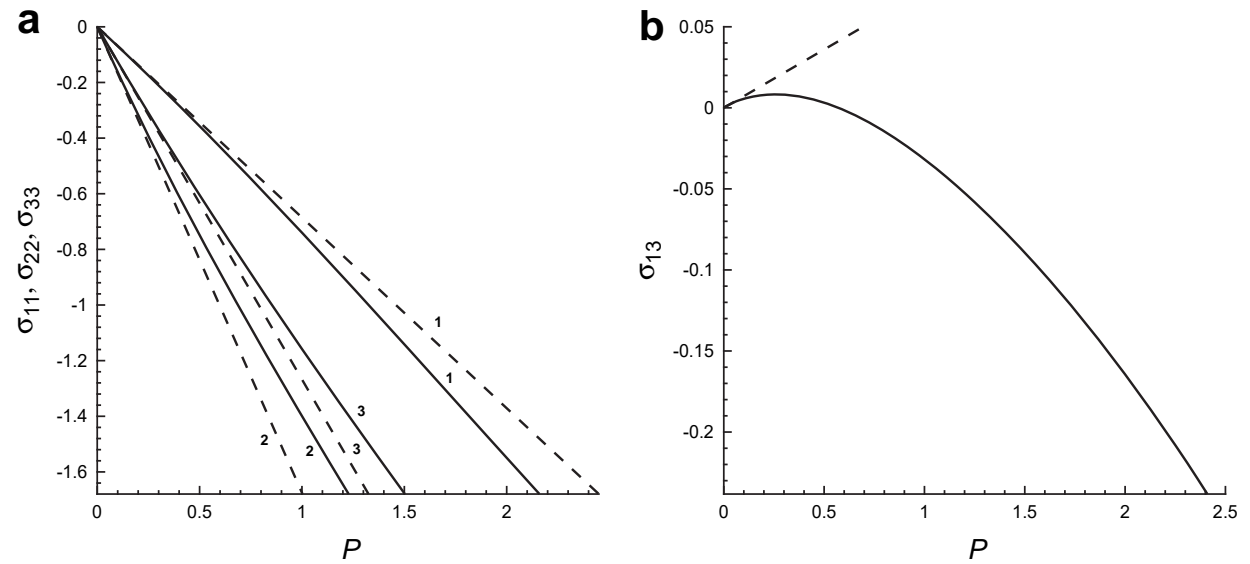

Fig. 10. (a) Plots of $\sigma_{11}, \sigma_{22}, \sigma_{33}$ (labelled $1,2,3$, respectively) versus $P$ for a tube with $A / B=0.5, L / B=5$ at point $(R, Z)=(0.75,4.5)$. (b) Plots of $\sigma_{13}$ versus $P$. Linear (dashed lines); nonlinear (solid curves).
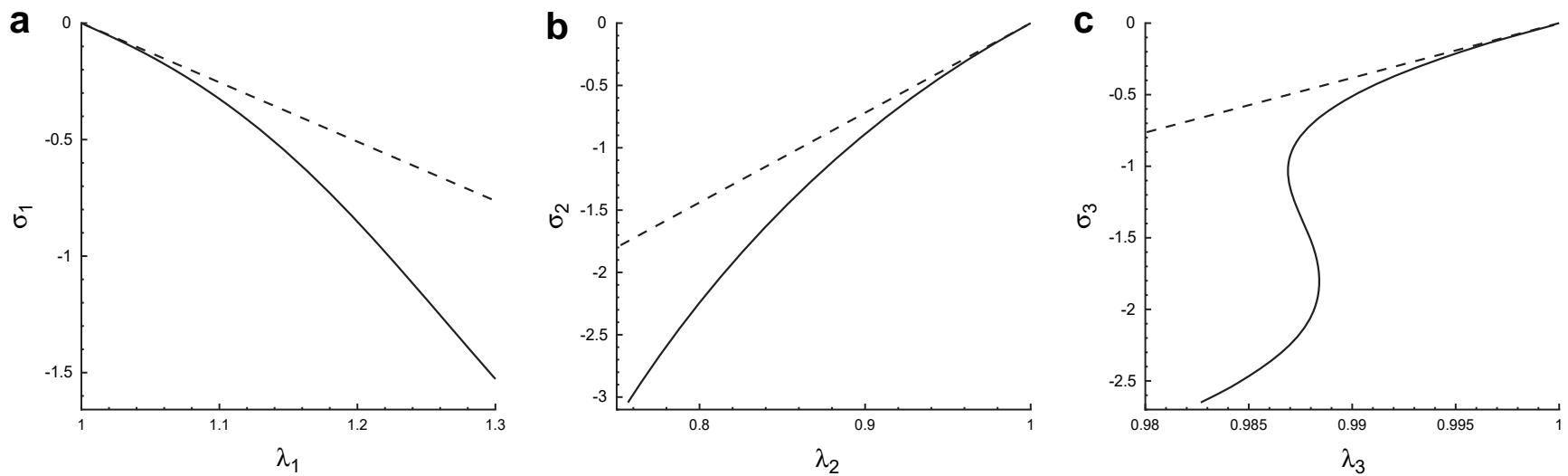

Fig. 11. Plots of the principal stresses versus the corresponding principal stretches for a tube with $A / B=0.5, L / B=5$, at the point $(R, Z)=(0.75,4.5)$ : nonlinear case. (a) $\sigma_{1}$ versus $\lambda_{1}$, (b) $\sigma_{2}$ versus $\lambda_{2}$, (c) $\sigma_{3}$ versus $\lambda_{3}$.

a

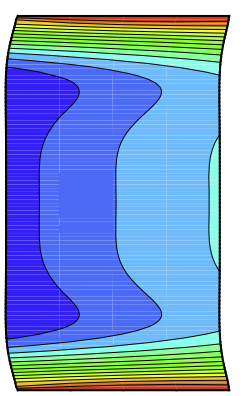

b

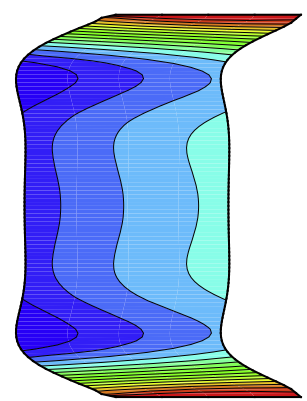

C

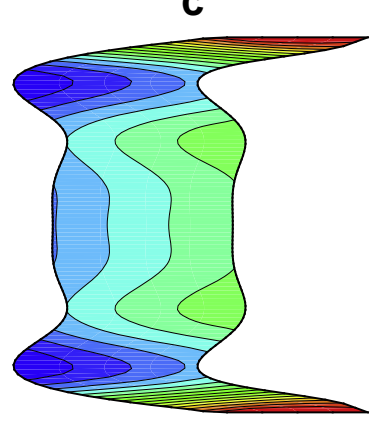

d

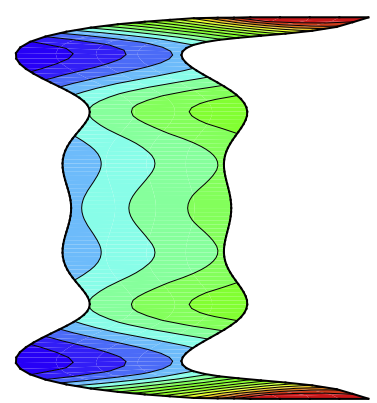

Fig. 12. (Not to scale) Nonlinear modes of deformation for a tube with $A / B=0.8, L / B=5$ : (a) mode- 1 , at $P=0.01$, (b) mode-2, at $P=0.11$, (c) mode-3, at $P=0.41$, (d) mode- 4 , at $P=0.61$. The contours shown are for the radial displacement $u$ superimposed on the $(r, z)$ deformed profile.

bulging at the corners at all for this case, as a result of which there is no shear splitting zone towards the ends, although the shear zone adjacent to the boundary region changes its sign, presumably due to the mode-2 deformation.

\subsection{Thinner and longer tubes: $A / B=0.8$ and $L / B=5$}

For longer and thinner tubes with $A / B=0.8$ and $L / B=5$, the most interesting feature is the occurrence of higher modes (multiple humps in the deformation) in the nonlinear case. Four modes from mode- 1 to mode- 4 are observed as the external pressure $P$ increases from 0 to about 0.66 , as shown in Fig. 12 . Mode-1 occurs for $0<P \leqq 0.01$, transitions to mode- 2 for $0.01 \leqq P \leq 0.16$, to mode-3 for $0.16 \leqq P \leq 0.41$ and mode- 4 for $0.41 \lesssim P \leqq 0.66$. For larger $P$ modes 5,6 and 7 were obtained, although the solution for large $P$ that gives rise to the higher modes is more demanding on the mesh qualities. No higher modes except mode- 2 were found for the linear model. 

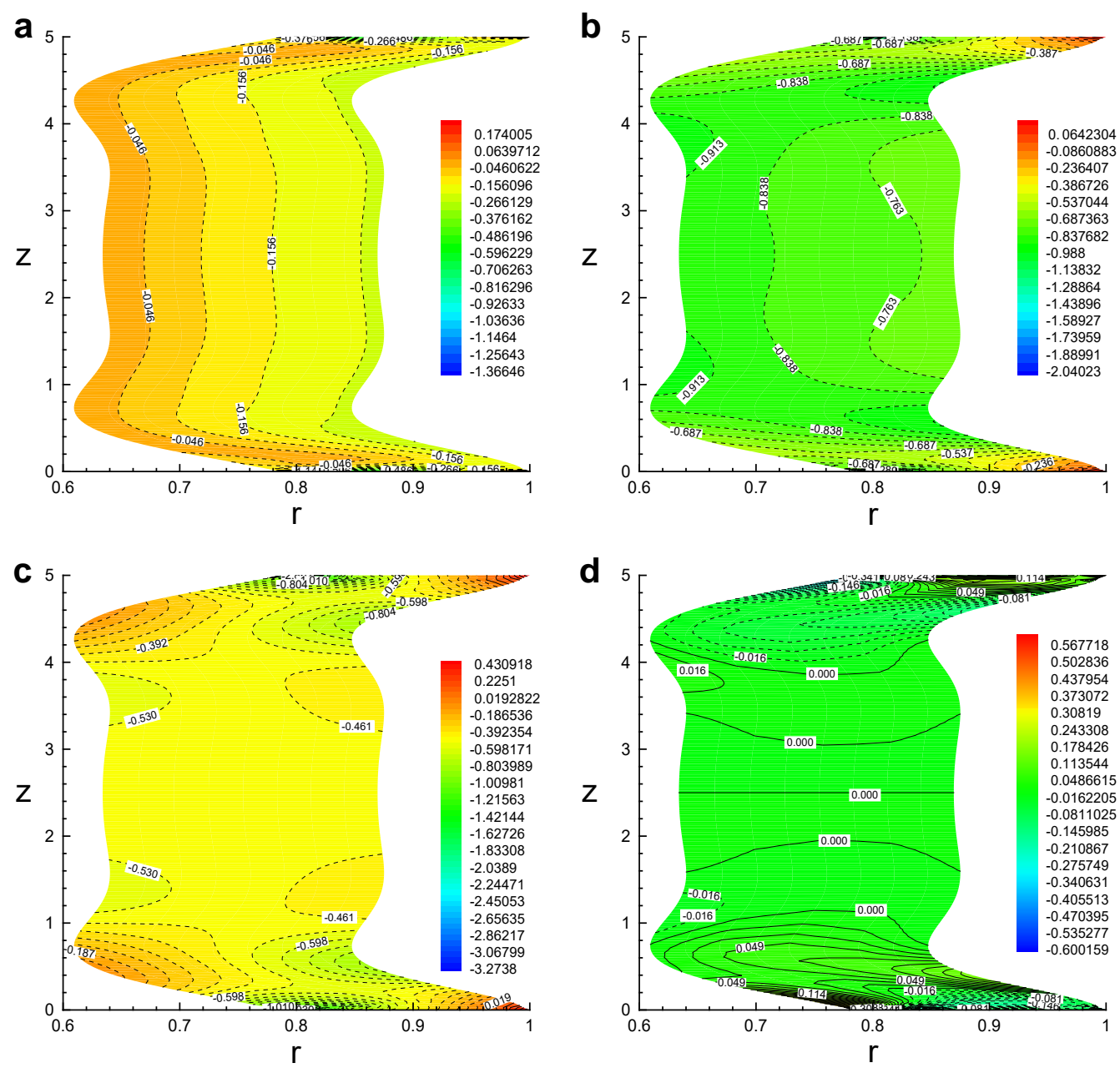

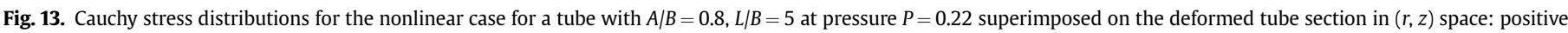
values are indicated by solid contours and negative values by dashed contours. (a) $\sigma_{11}$, (b) $\sigma_{22}$, (c) $\sigma_{33}$, (d) $\sigma_{13}$.

Fig. 13 shows the distributions of all the Cauchy stress components for the nonlinear case at $P=0.22$. Again, there are two major differences when compared with the corresponding linear case (not shown). One is that the nonlinear model presents a higher mode (mode- 4 in this case), where the corresponding linear case exhibits only mode- 2 . The other is the shear splitting pattern in the nonlinear model, which expands from the two ends towards the middle section. Although this is not shown here we note that the boundary effect is more limited to near the two ends in the linear model, with the same sign of $\sigma_{13} \geq 0$ near the upper end, and $\sigma_{13} \leq 0$ near the lower end. The patterns of $\sigma_{22}$ and $\sigma_{33}$ are also quite interesting, with the nonlinear effects more clearly focused on the boundaries.

\section{Discussion and conclusions}

We have derived the general partial differential equations in Lagrangian form governing the large axisymmetric deformations of a thick-walled tube composed of incompressible isotropic elastic material, without any assumptions limiting the magnitude of the deformation or material nonlinearity. Comparison has been made with the corresponding linear model for tubes with different wall thickness and length ratios.

For small deformations the linear and nonlinear models give very similar results. However, the predictions of the linear and nonlinear models are very different under large external pressure, and the dominant nonlinear features are the corner bulging, and, for longer tubes, the occurrence of higher modes of deformation. Note, however, that the higher modes for longer and thinner tubes can be associated with geometrical nonlinearity and are not features unique to material nonlinearity (Heil and Pedley, 1995; Heil, 1996). Although we don't distinguish the material and geometric nonlinearities in the present study, we have observed that material nonlinearity is more important in the shorter and thicker tubes, for which the strains computed are larger, while geometrical nonlinearity seems to dominate in the longer and thinner tubes, for which the strains are much smaller. The Cauchy stresses, especially the shear stress, exhibit the greatest differences between the predictions of the linear and nonlinear theories. Shear splitting, with alternating signs of the shear stress in different regions is a unique nonlinear feature. As a result, the end boundary constraints have a much stronger influence on the deformation and stresses in the rest of the tube for the nonlinear model. This is the first systematic nonlinear treatment of externally pressurized thick-walled elastic tubes, albeit using the simple neo-Hookean material, and the results may have significant implications for certain physiological applications involving soft vessels undergoing large deformation.

The nonlinear system of equations has been solved by using the $\mathrm{C}++$ based finite element package Libmesh. It should be 
noted that due to the complex nature of the nonlinear equations, it was extremely difficult to obtain converged solutions numerically using approximate Newton solvers and it was necessary to derive the Jacobian matrix $\mathbf{J}$ analytically, and to use the corresponding linear solution as an initial solution in order to obtain convergence. In addition, since the geometry of the tube in the reference configuration and the boundary conditions and external pressure condition are all symmetric about the midplane of the tube, a symmetric mesh needs to be used to achieve perfectly symmetric solutions.

We have noted that the nonlinear effects for long, thin tubes are limited to a layer of width $\sqrt{(B-A) A}$ near the boundaries; see Fig. 12. This agrees broadly with the examples given by Libai and Simmonds (1998) on the behaviour of nonlinear shell-membrane materials. No direct results can be found in (Libai and Simmonds, 1998) for a neo-Hookean cylindrical shell under external pressure. However, qualitative comparison is possible with the results of Tait et al. (1996) who studied the deformation of a neo-Hookean cylindrical membrane under twist. They found that at larger values of the prescribed twist, wrinkling occurs in the interior and the membrane remains tense near the boundaries. Although these are obtained for different boundary conditions, the nonlinear effects such as the presence of the boundary layer and multi-modes are similar to these shown in Fig. 12.

Since we have dealt with axisymmetric problems, we can only simulate the necked or barreled states of a cylinder. In addition, we have not carried out any bifurcation analysis and it remains to ascertain the stability status of the solutions obtained, although previous studies on similar nonlinear problems, albeit with different boundary conditions (Negrón-Marrero, 1999), have shown that there exist nontrivial axisymmetric stable (half neck or multiple-neck) solutions. However, in many physiological situations, nonlinear deformations that break this symmetry (both for the original deformation or the bifurcation analysis) could be more significant. We shall pursue this problem in subsequent work.

\section{Acknowledgements}

Yunfei Zhu is supported by a UK ORS award and by a scholarship from the Faculty of Information and Mathematical Science at Glasgow University. We are also grateful for Libmesh developers at the University of Texas and Dr. Boyce Griffith at New York University for their help with using Libmesh.

\section{References}

Anlike, M., Moritz, W.E., Ogden, E., 1968. Transmission characteristics of axial waves in blood vessels. Journal of Biomechanics 1, 235-246.
Balay, S., Buschelman, K., Eijkhout, V., Gropp, W.D., Kaushik, D., Knepley, M.G., McInnes, L.C., Smith, B.F., Zhang, H., 2008. PETSc Users Manual. Technical Report ANL-95/11 - Revision 3.0.0. Argonne National Laboratory.

Beatty, M.F., Dadras, P., 1976. Some experiments on the elastic stability of some highly elastic bodies. International Journal of Engineering Science 14, 233-238.

Bertram, C.D., 1982. Two modes of instability in a thick-walled collapsible tube conveying a flow. Journal of Biomechanics 15, 223-224.

Bertram, C.D., 1987. The effects of wall thickness, axial strain and end proximity on the pressure-area relation of collapsible tubes. Journal of Biomechanics 20, 863-876.

Bertram, C.D., Raymond, C.J., Pedley, T.J., 1990. Mapping of instabilities for flow through collapsed tubes of differing length. Journal of Fluids and Structures 4, 125-153.

Demiray, H., 1996. Nonlinear waves in a prestressed thick elastic tube filled with an invisid fluid. International Journal of Engineering Science 34, 1519-1530.

Demiray, H., 1998. Nonlinear wave modulation in a fluid filled thick elastic tube. International Journal of Engineering Science 36, 1061-1082.

Erbay, H.A., Demiray, H., 1995. Finite axisymmetric deformations of elastic tubes: an approximate method. Journal of Engineering Mathematics 29, 451-472.

Green, A.E., Rivlin, R.S., Shield, R.T., 1952. General theory of small elastic deformations superposed on finite elastic deformations. Proceedings of the Royal Society of London A 211, 128-154.

Haughton, D.M., Ogden, R.W., 1979. Bifurcation of inflated circular cylinders of elastic material under axial loading-II, exact theory for thick-walled tubes. Journal of the Mechanics and Physics of Solids 27, 489-512.

Heil, M., 1996. The stability of cylindrical shells conveying viscous flow. Journal of Fluids and Structures 10, 173-196.

Heil, M., Pedley, T.J., 1995. Large axisymmetric deformation of a cylindrical shell conveying a viscous flow. Journal of Fluids and Structures 9, 237-256.

Kirk, B., Peterson, J.W., Stogner, R.H., Carey, G.F., 2006. LibMesh: a C++ library for parallel adaptive mesh refinement/coarsening simulations. Engineering with Computers 22, 237-254.

Libai, A., Simmonds, J.G., 1998. The Nonlinear Theory of Elastic Shells. Cambridge University Press.

Marzo, A., Luo, X.Y., Bertram, C.D., 2005. Three-dimensional collapse and steady flow in thick-walled flexible tubes. Journal of Fluids and Structures 20, 817-835.

Moodie, T.B., Swaters, G.E., 1989. Nolinear waves and shock calculations for a hyperelastic fluid-filled tube. Quarterly Applied Mathematics 47, 705732.

Negrón-Marrero, P.V., 1999. An analysis of the linearized equations for axisymmetric deformations of hyperelastic cylinders. Mathematics and Mechanics of Solids 4, 109-133.

Nowinski, J.L., Shahinpoor, M., 1969. Stability of an elastic circular tube of arbitrary wall thickness subjected to an external pressure. International Journal of NonLinear Mechanics 4, 143-158.

Ogden, R.W., 1997. Non-linear Elastic Deformations. Dover Publications, New York.

Rudinger, G., 1970. Shock waves in a mathematical model of the aorta. Journal of Applied Mechanics 37, 34-37.

Tait, R.J., Steigmann, D.J., Zhong, J.L., 1996. Finite twist and extension of a cylindrical elastic membrane. Acta Mechanica 117 (1), 129-143.

Wang, A.S.D., Ertepinar, A., 1972. Stability and vibrations of elastic thick-walled cylindrical and spherical shells subjected to pressure. International Journal of Non-Linear Mechanics 7, 539-555.

Yamaki, N., 1969. Buckling of circular cylindrical shells under external pressure. Reports of the Institute of High Speed Mechanics 20, 35-55.

Yamaki, N., 1984. Elastic Stability of Circular Cylindrical Shells. North-Holland, Amsterdam.

Zhu, Y., Luo, X.Y., Ogden, R.W., 2008. Asymmetric bifurcations of thick-walled circular cylindrical elastic tubes under axial loading and external pressure. International Journal of Solids and Structures 45, 3410-3429. 